\title{
Job Satisfaction among Care Aides in Residential Long-Term Care: A Systematic Review of Contributing Factors, Both Individual and Organizational
}

\author{
Janet E. Squires, ${ }^{1,2}$ Matthias Hoben, ${ }^{3}$ Stefanie Linklater, ${ }^{2}$ Heather L. Carleton, ${ }^{3}$ \\ Nicole Graham, ${ }^{1}$ and Carole A. Estabrooks ${ }^{3}$ \\ ${ }^{1}$ School of Nursing, University of Ottawa, 451 Smyth Road, Ottawa, ON, Canada K1H 8M5 \\ ${ }^{2}$ Ottawa Hospital Research Institute, Centre for Practice-Changing Research (CPCR), 501 Smyth Road, Room 1282, Box 711, \\ Ottawa, ON, Canada K1H $8 L 6$ \\ ${ }^{3}$ Faculty of Nursing, University of Alberta, Level 3, Edmonton Clinic Health Academy, 1140587 Avenue NW Edmonton, \\ AB, Canada T6G 1C9
}

Correspondence should be addressed to Janet E. Squires; jasquires@ohri.ca

Received 25 March 2015; Accepted 25 June 2015

Academic Editor: Maria H. F. Grypdonck

Copyright (C) 2015 Janet E. Squires et al. This is an open access article distributed under the Creative Commons Attribution License, which permits unrestricted use, distribution, and reproduction in any medium, provided the original work is properly cited.

Despite an increasing literature on professional nurses' job satisfaction, job satisfaction by nonprofessional nursing care providers and, in particular, in residential long-term care facilities, is sparsely described. The purpose of this study was to systematically review the evidence on which factors (individual and organizational) are associated with job satisfaction among care aides, nurse aides, and nursing assistants, who provide the majority of direct resident care, in residential long-term care facilities. Nine online databases were searched. Two authors independently screened, and extracted data and assessed the included publications for methodological quality. Decision rules were developed a priori to draw conclusions on which factors are important to care aide job satisfaction. Forty-two publications were included. Individual factors found to be important were empowerment and autonomy. Six additional individual factors were found to be not important: age, ethnicity, gender, education level, attending specialized training, and years of experience. Organizational factors found to be important were facility resources and workload. Two additional factors were found to be not important: satisfaction with salary/benefits and job performance. Factors important to care aide job satisfaction differ from those reported among hospital nurses, supporting the need for different strategies to improve care aide job satisfaction in residential long-term care.

\section{Background}

1.1. Aging and Residential Long-Term Care. In the first half of the 21st century, the global population 60 years or over is projected to expand threefold to nearly 2 billion, with 33 countries having more than 10 million people 60 years of age or over [1]. With this dramatic demographic shift come sharp increases in numbers of older adults with agerelated dementias (ARDs) [2-4]. ARDs are a world-wide public health concern, with nearly 7.7 million new cases globally each year [5]. ARDs are the main factor escalating the need for residential long-term care (LTC) [6-8]. Without dramatic breakthroughs in ARD prevention, treatment, or management, the need for residential LTC facilities will increase as the population ages.

Residential LTC facilities offer 24-hour on-site housing and health care services to the elderly, defined as persons of age 65 and older. The individuals cared for at these facilities are frail, vulnerable, functionally dependent older adults who frequently suffer from a range of chronic diseases or disabilities $[9,10]$. There are varying terms to describe residential LTC facilities, for example, residential care, assisted living facilities, nursing homes, long-term care homes, and residential aged care. These facilities offer different levels of care and may be individual structures or associated within or with community care centres or hospitals. 
Nonprofessional workers (care aides, also commonly referred to as personal care workers, nursing assistants, and nurse aides) provide most direct nursing care in these facilities. In Canada and the USA, these individuals provide 70$80 \%$ of direct care to residents in residential LTC facilities [1115]. These workers often lack adequate formal qualifications [16], continuing education, and monitoring [16], which poses a serious concern in meeting minimum standards of care in LTC [17]. Care aide duties may include apparently simple tasks related to personal hygiene, toileting, feeding, and housekeeping, but aides are also vital to the quality of life of our growing vulnerable older adult population.

Across all care settings, we currently see widespread shortages of all levels of nursing care providers and high turnover rates. This global issue is increasingly important to both developed and developing countries [18-21] and of increasing concern in many countries $[17,18]$ and the LTC sector. Staff turnover in residential long-term care facilities ranges from $40 \%$ to $500 \%$ [22, 23]. Numerous factors have been linked to turnover of nursing care providers; job satisfaction however is by far the most frequently cited [2426].

1.2. Job Satisfaction. Multiple definitions of job satisfaction abound in the literature. For this review, we defined job satisfaction using the traditional model frequently cited in empirical studies of job satisfaction of nursing care providers. This model focuses on job satisfaction as the affective orientation of an employee towards his or her work (i.e., on the feelings an individual has about his or her job [20, 27]). This "affective" focus can be seen in frequently cited definitions of job satisfaction scholars such as Locke $[28,29]$ who describes job satisfaction as a "pleasurable or positive emotional state resulting from the appraisal of one's job or job experiences" and Brooke and colleagues who describe job satisfaction as "an affective response to the job situation" [30].

Not all dissatisfied staff will leave their job, but dissatisfaction may impact their work, their coworkers, and the quality of resident care delivered. Dissatisfied staff often show signs of an unreliable work ethic, such as tardiness and taking unscheduled days off [31]. Some dissatisfied staff show greater aggression towards other workers [32] and residents [33]. Job dissatisfaction is associated with reduced quality of resident care [34] and resident quality of life [35], as well as reduced ability of organizations to change [36]. Conversely, caregivers (including care aides) who report perceiving high quality of care in their facilities also report higher satisfaction with their job [31]. For the last several decades, quality of care in some residential LTC facilities has been consistently reported as substandard [37-39]. This fact, coupled with evidence of residential LTC facilities' limited ability to change in a meaningful way [31], highlights the importance of understanding job satisfaction of care aides in these facilities.

While multiple individual studies examine factors related to care aide's job satisfaction and/or job satisfaction in residential LTC facilities, this evidence has not yet been synthesized. However, a synthesis of factors associated with job satisfaction among hospital registered nurses was recently published. In that review, Lu and colleagues [20] found that job satisfaction is closely related to working conditions and the organizational environment, job stress, role conflict and ambiguity, role perception and role content, and organizational and professional commitment [20]. The purpose of this systematic review is to synthesize the evidence on factors (both individual and organizational) associated with job satisfaction among care aides in residential LTC facilities.

\section{Methods}

2.1. Selection Criteria for Types of Studies. Primary studies that used experimental (randomized controlled trials, clinical trials, and quasi-experimental, e.g., pre/posttest [40]) and nonexperimental (observational and qualitative [40]) designs examining factors associated with job satisfaction for care aides in residential LTC facilities were eligible for inclusion. Studies were limited to those published in English, with no restrictions on country of origin or publication date.

2.2. Selection Criteria for Types of Participants, Factors, and Outcomes. Participants included care aides, nurse aides, and/or nursing assistants. Care aide was defined as a nonprofessional worker providing direct resident care, under the supervision of a registered nurse (RN) or licensed practical nurse (LPN) or registered practical nurse (RPN). Nursing assistant/aide (NA) was defined as a person who has completed a brief health care training program and who provides support services for RNs and LPNs/RPNs. An NA is termed a certified nurse aide (CNA) when certified by a state agency (USA) or province (Canada) [41]. Factors (independent variables) of interest were any individual or organizational variable associated with job satisfaction, the outcome of interest. We defined job satisfaction as the affective orientation of an employee towards his or her work (i.e., on the feelings an individual has about his or her job $[20,27])$. We included studies of job satisfaction that met this definition. We included studies of job satisfaction in other care providers only if a separate analysis of care aide/NA job satisfaction was provided or could be extracted. Only studies published in English were eligible for inclusion.

2.3. Search Strategy for Identification of Studies. The search strategy (Table 1) was developed in consultation with a health sciences librarian. We searched nine online databases: the Cochrane Database of Systematic Reviews, CINAHL, Business Source Complete, Medline, EMBASE, AARP AgeLine, Web of Science, SCOPUS, and ABI Inform. Key words included long-term care, care aide, and job satisfaction (and their synonyms).

2.4. Study Identification. Two team members independently screened all abstracts identified by the search strategy $(n=$ 967 after removal of duplicates). Full text copies were retrieved for all citations identified as potentially relevant to our review aim or with insufficient information to make a decision on relevance $(n=164)$. Any article not meeting all inclusion criteria outlined above was excluded from the review. 
TABLE 1: Search strategy (all searches performed through to May 1, 2013).

\begin{tabular}{|c|c|}
\hline Database & Search terms \\
\hline CINAHL & $\begin{array}{l}\text { (MH "Nursing Assistants") OR (MH "Nursing Home Personnel") } \\
\text { "health care aide*” or "nursing assistant"” or "nurs* aide*” or "personal care aide*” or "resident companion*” or } \\
\text { "geriatric aide*” or hca } \\
\text { (MH “Job Satisfaction") } \\
\text { ("Job satisfaction") or career N2 satisf* or work N2 satisf* or employ* N2 satisf" }\end{array}$ \\
\hline $\begin{array}{l}\text { Business Source } \\
\text { Complete }\end{array}$ & 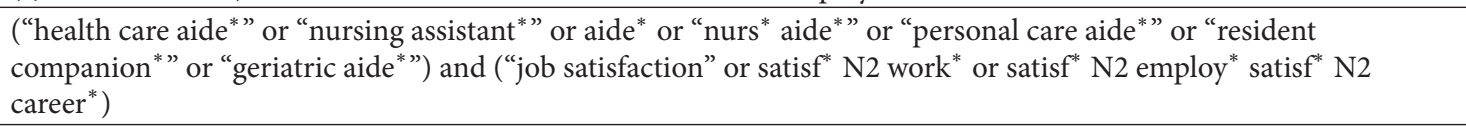 \\
\hline Medline & 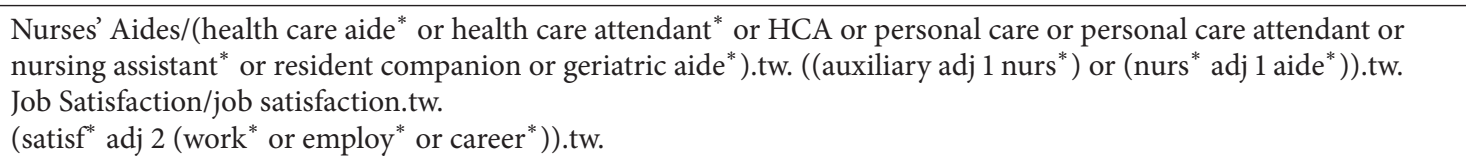 \\
\hline EMBASE & $\begin{array}{l}\text { nursing assistant/(health care aide }{ }^{*} \text { or health care attendant }{ }^{*} \text { or HCA or personal care or personal care attendant or } \\
\left.\text { nursing assistant }{ }^{*} \text { or resident companion or geriatric aide }{ }^{*}\right) . t w . \\
\left(\left(\text { auxiliary adj } 1 \text { nurs }{ }^{*} \text { ) or (nurs* adj } 1 \text { aide }^{*}\right)\right) . t w . \\
\text { job satisfaction/job satisfaction.tw. } \\
\left(\text { satisf }^{*} \text { adj } 2\left(\text { work }^{*} \text { or employ }{ }^{*} \text { or career* }\right)\right) . t w .\end{array}$ \\
\hline AARP Ageline & $\begin{array}{l}\text { "Nurses-Aides".de. } \\
\text { (health care aide* or health care attendant* or HCA or personal care or personal care attendant or nursing assistant* } \\
\text { or resident companion or geriatric aide*).tw. } \\
\left((\text { auxiliary adj } 1 \text { nurs* }) \text { or }\left(\text { nurs* adj } 1 \text { aide }^{*}\right)\right) . t w . \\
\text { "Job-Satisfaction".de. } \\
\text { job satisfaction.tw. } \\
\text { (satisf* adj } 2 \text { (work* or employ* or career*)).tw. }\end{array}$ \\
\hline Web of Science & $\begin{array}{l}\text { TS }=\text { ("health care aide" " or "health care attendant" or HCA or "personal care attendant " or "nursing assistant " or } \\
\text { "resident companion*” or "geriatric aide*”) AND TS = ("job satisfaction") } \\
\text { Databases = SCI-EXPANDED, SSCI, CPCI-S }\end{array}$ \\
\hline SCOPUS & $\begin{array}{l}\text { (TITLE-ABS-KEY(“health care aide*” OR "health care attendant*” OR hca OR "personal care attendant*” OR } \\
\text { "nursing assistant" OR "resident companion" " OR "geriatric aide" }{ }^{*} \text { ) AND TITLE-ABS-KEY(“job satisfaction”)) }\end{array}$ \\
\hline ABI Inform & $\begin{array}{l}\text { ("health care aide"” OR "nursing assistant"” OR "nurs" aide"” OR "personal care aide*” OR "resident companion"” } \\
\text { OR "geriatric aide"”) AND ("job satisfaction") }\end{array}$ \\
\hline Cochrane & job satisfaction \\
\hline
\end{tabular}

Two reviewers independently assessed all retrieved articles; 42 articles were retained (see PRISMA flow diagram in Figure 1). Screening discrepancies were resolved through consensus.

2.5. Quality Assessment. Methodological quality of the final set of included articles was independently assessed by two reviewers with disagreements resolved through consensus. Four previously validated assessment tools were used. Quantitative studies were assessed using 1 of 3 tools: (1) the Quality Assessment and Validity Tool for Cross-Sectional Studies, (2) the Quality Assessment and Validity Tool for Pre/Posttest Studies, and (3) the Quality Assessment Tool for Quantitative Studies (used for randomized controlled trials). The original tools are described in detail in previously published systematic reviews (e.g., [42-45]). Quality assessment considered appropriateness of study design based on the research objectives, sample, measurement of key variables (individual and organizational factors) and the outcome of interest (job satisfaction), and appropriateness of the statistical analysis.

The first two tools, the Quality Assessment and Validity Tool for Cross-Sectional Studies and the Quality Assessment and Validity Tool for Pre/Posttest Studies, were originally developed by members of our team based on Cochrane guidelines (in existence since 2001) and the medical literature $[86,87]$ and have been used in other published systematic reviews by our group [42-45]. The cross-sectional tool contains a maximum of 16 points and assesses studies in three core areas: sampling, measurement, and statistical analysis. The pre/posttest tool contains a maximum of 18 points and assesses studies in 6 core areas: sampling, design, control of confounders, data collection and outcome measurement, statistical analysis, and dropout. To derive a final quality score for each article, we divided the total points scored by the total points possible (16 or 18 minus the number of points not applicable for the article). Each study was then classified as weak $(\leq 0.50)$, moderate-weak $(0.51$ to 0.65$)$, moderatestrong $(0.66$ to 0.79$)$, or strong $(\geq 0.80)$. This rating system has been used in several recent reviews [43-45] and is based on a scoring system developed by De Vet et al. [88]. These two tools were used to assess the methodological quality of all cross-sectional $(n=29)$ and pre/post $(n=7)$ studies included in our review.

The third quality assessment tool used in this review was the Quality Assessment Tool for Quantitative Studies, developed by the Effective Public Health Practice Project, 


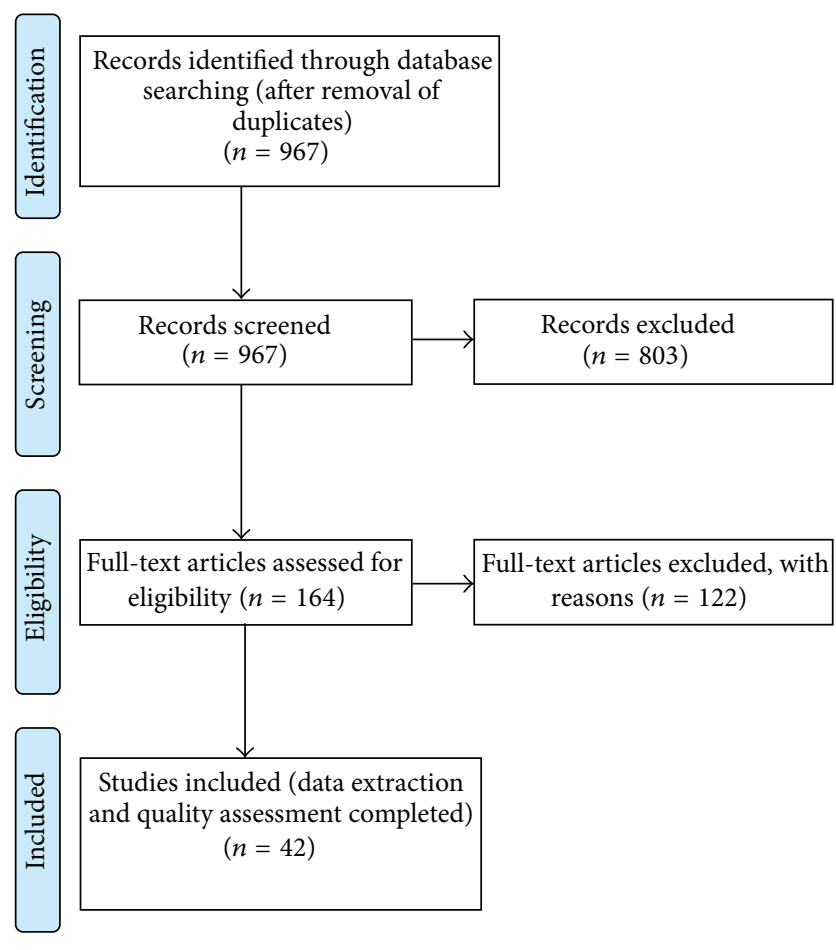

Figure 1: PRISMA flow diagram.

Canada. This tool has been judged suitable to be used in systematic reviews of effectiveness (measuring interventions) [89] and been shown to have content and construct validity [90]. The tool assesses studies on the basis of six areas: selection bias, study design, confounders, blinding, data collection methods, and withdrawals/dropouts. Each article is scored as weak, moderate, or strong in each of these areas according to preset criteria within the tool. The tool developers do not provide a means for calculating an overall quality score. However, in order to compare the quality scores for the included articles assessed with this tool to those that used cross-sectional and pre/posttest tools, we derived an overall quality score. We applied the scoring system of this tool used in a previously published review [44]. This score was derived by assigning values of 1,2 , and 3 to the categorizations of weak, moderate, and strong respectively. A final quality score was then obtained by dividing the summative score obtained by the total amount of points possible. Each study was classified as weak (1 to 1.5), moderate-weak (1.6 to 2.0), moderate-strong (2.1 to 2.5 ), or strong ( $>2.5$ ) by applying the same categorization system used (and published) in the crosssectional and pre/posttest tools. The Quality Assessment Tool for Quantitative Studies Tool was used to assess RCT studies included in this review $(n=1)$.

Qualitative studies were assessed using the Critical Appraisal Skills Programme (CASP) Quality Assessment Tool [91]. This tool assesses qualitative studies through 10 questions on research aims, appropriateness of research design, appropriateness of recruitment strategy, data collection, relationship between researcher and participants, ethical issues, data analysis, statement of findings, and value of the research [91]. A final quality score for each article was then obtained by dividing the summative score obtained by the total amount of points possible. Each study was classified using the same rating scale as for the cross-sectional and pre/posttest studies: weak $(\leq 0.50)$, moderate-weak ( 0.51 to 0.65$)$, moderate-strong (0.66 to 0.79$)$, or strong $(\geq 0.80)$.

2.6. Data Extraction and Synthesis. One team member extracted data from all included articles, double-checked by a second team member for accuracy. Discrepancies in data extraction were resolved through consensus. Data were extracted on year of publication, title, journal, country of origin, purpose/objectives, data collection methods, study design, sample size and setting, job satisfaction measure (including number of items, reliability, and validity), independent variables investigated (individual and organizational factors), analyses, and main outcome(s).

Data on individual factors were grouped into five broad categories (each having subgroups). The five broad categories were (1) sociodemographic, (2) education, (3) healthcare provider characteristics, (4) personal life, and (5) other. Data on organizational factors were also grouped into five categories, again with subgroups. The five broad organizational categories were (1) facility, (2) work environment, (3) supervision, (4) staffing, and (5) other. Categories (and their subgroups) were not predetermined; after reviewing and extracting data, we found that factors relevant to our aim centered on these themes. We used the primary studies authors' conceptualizations in this grouping. For example, if an author reported investigating autonomy, it was classified as autonomy in our synthesis; we did not reclassify any variables based on the definitions provided in the primary studies. Grouping the factors facilitated comparing and interpreting their importance to care aide job satisfaction in residential LTC facilities.

We used a vote-counting approach to synthesize the quantitative evidence. The overall assessment of a relationship between a factor and job satisfaction was based on the percentage of studies demonstrating, or failing to demonstrate, statistically significant associations. As recommended by Grimshaw et al. [92], we supplemented this by extracting direction and magnitude of effect for all factors displaying statistically significant effects $(p<0.05)$, where provided. If a study included multiple analyses (e.g., univariate, bivariate, and/or multivariate), we relied on the highest level model (e.g., multivariate where available).

Qualitative findings were assessed for themes and summarized narratively. We applied the following previously published a priori rules [45] to guide our quantitative synthesis.

(1) To conclude whether or not a factor (individual or organizational) was associated with job satisfaction, it had to be assessed four or more times (this could reflect two assessments of different variables comprising the same factor from one study). If a factor was assessed fewer than 4 times it was coded as inconsistent (i.e., insufficient evidence to reach a conclusion). 
(2) Factors assessed four or more times were coded as

(a) significant with (important to) job satisfaction if $60 \%$ or more of the quantitative tests showed a significant association between the factor and job satisfaction;

(b) nonsignificant with (not important to) job satisfaction if $60 \%$ or more of the quantitative tests showed a nonsignificant association between the factor and job satisfaction;

(c) equivocal with (undetermined importance to) job satisfaction if $<60 \%$ of the quantitative tests showed significant/nonsignificant associations between the factor and job satisfaction.

\section{Results}

3.1. Description of Studies. Forty-two studies were included in the review. The majority $(n=29)$ of studies used a cross-sectional survey design $[9,10,33,46-48,52-54,56-$ $58,60-62,64,66-71,73-75,77-79,93]$. Of these, 1 study used mixed methods (survey plus qualitative data) [78], 1 study used a randomized controlled trial [59], and 7 studies used observational before-and-after quasi-experimental (pre/posttest) design $[49,51,55,65,72,76,80]$. One of the quasi-experimental studies also used mixed methods and included qualitative data [55]. Five additional studies used a qualitative design [81-85]. Overall, our sample included 37 studies with quantitative statistical data and 7 studies with qualitative data.

Studies were conducted with CNAs $(n=24)$, NAs $(n=7)$, and care aides $(n=5) ; 6$ studies included multiple groups. Studies were set in residential LTC facilities $(n=22)$, other LTC facilities $(n=7)$, assisted living facilities $(n=1)$, skilled nursing facilities $(n=2)$, and combined assisted living/skilled nursing facilities $(n=1)$. Countries of origin were the USA $(n=37)$, Taiwan $(n=2)$, Sweden $(n=1)$, Canada $(n=1)$, and Australia $(n=1)$. Studies were published between 1976 and 2012 with the majority being published after $2000(n=$ 28). Different measures of job satisfaction were used across the studies. Only 4 job satisfaction tools were used in greater than one study: Minnesota Satisfaction Questionnaire $(n=4$ studies), Job Descriptive Index ( $n=3$ studies), Benjamin Rose Institute Job Satisfaction Scale ( $n=3$ studies), Job Diagnostic Survey ( $n=3$ studies), and Job Attitude Scale ( $n=$ 2 studies). Details on included studies are given in Table 2; a list of studies was excluded and the reason(s) for their exclusion are in Additional File 1 (in Supplementary Material available online at http://dx.doi.org/10.1155/2015/157924).

3.2. Methodological Quality of Included Studies. We completed 44 quality assessments on the 42 included studies; the 2 studies $[55,78]$ with mixed methods designs both had 2 quality assessments done. Details of methodological quality assessments of all 42 studies are in Additional File 2.

From the 44 quality assessments, 5 (11\%) studies were rated strong $[9,78,81,84,85], 6(14 \%)$ high moderate $[53,56$, 66-68, 73], 15 (34\%) low moderate [10, 52, 57, 58, 61, 64, 65,
$69,71,72,75,76,78,83,93]$, and $18(41 \%)$ weak $[33,46-49,51$, $54,55,59,60,62,70,74,77,79,80,82]$. Differences in quality assessment arose mainly from sample representativeness, treatment of missing data, and appropriateness of statistical test(s) used.

We conducted a sensitivity analysis, comparing findings from all studies with those rated moderate and strong. No significant differences were noted; thus, we report findings from all studies.

\subsection{Individual/Organizational Factors and Job Satisfaction}

3.3.1. Quantitative Findings. A total of 33 and 25 studies investigated the statistical association of care aide job satisfaction with individual and organizational factors, respectively. Details of the statistical effects including direction of effect and significance of the studies meeting our criteria to be able to draw a conclusion (i.e., assessed four or more times) are presented in Table 3 (individual factors) and Table 4 (organizational factors). Additionally, an overall picture of the findings and the resulting conclusions drawn are depicted in Table 5 (individual factor conclusions) and Table 6 (organizational factor conclusions). A summary of findings with respect to the relationship between job satisfaction and individual and organizational factors that were assessed less than four times can be found in Additional File 3.

As illustrated in Tables 3 and 5, 11 individual (care aide) factors spanning 4 of the 5 main categories were assessed 4 or more times. Two of these factors, both under the category of healthcare provider characteristics, had a significant positive relationship with care aide job satisfaction: empowerment and autonomy. Six additional individual factors (spanning 3 categories; categories are identified in brackets) showed no relationship to job satisfaction: age (sociodemographics), ethnicity (sociodemographics), gender (sociodemographics), level of education/years of education (education), special training (education), and years of experience as a care aide (healthcare provider characteristics). The remaining 3 individual factors assessed 4 or more times showed equivocal findings in relation to care aide job satisfaction: current position (personal characteristics), employment status (personal characteristics), and stress (personal life).

Tables 4 and 6 depict the five organizational factors that were assessed 4 or more times; these 5 factors spanned 3 organizational categories. Two of these factors had a significant positive relationship overall with care aide job satisfaction (the categories are identified in brackets): resources (facility) and workload (workload). Two factors showed no relationship to job satisfaction: satisfaction with salary/benefits (work environment) and job performance (work environment). The remaining organizational factor assessed 4 or more times was support from coworkers (work environment) which had an equivocal relationship with care aide job satisfaction.

3.3.2. Qualitative Findings. Most factors identified in the qualitative data were organizational in nature and were reported in a single study (Table 7). Overall, qualitative findings support the conclusions drawn from the synthesis of the quantitative data. Factors related to work environment 


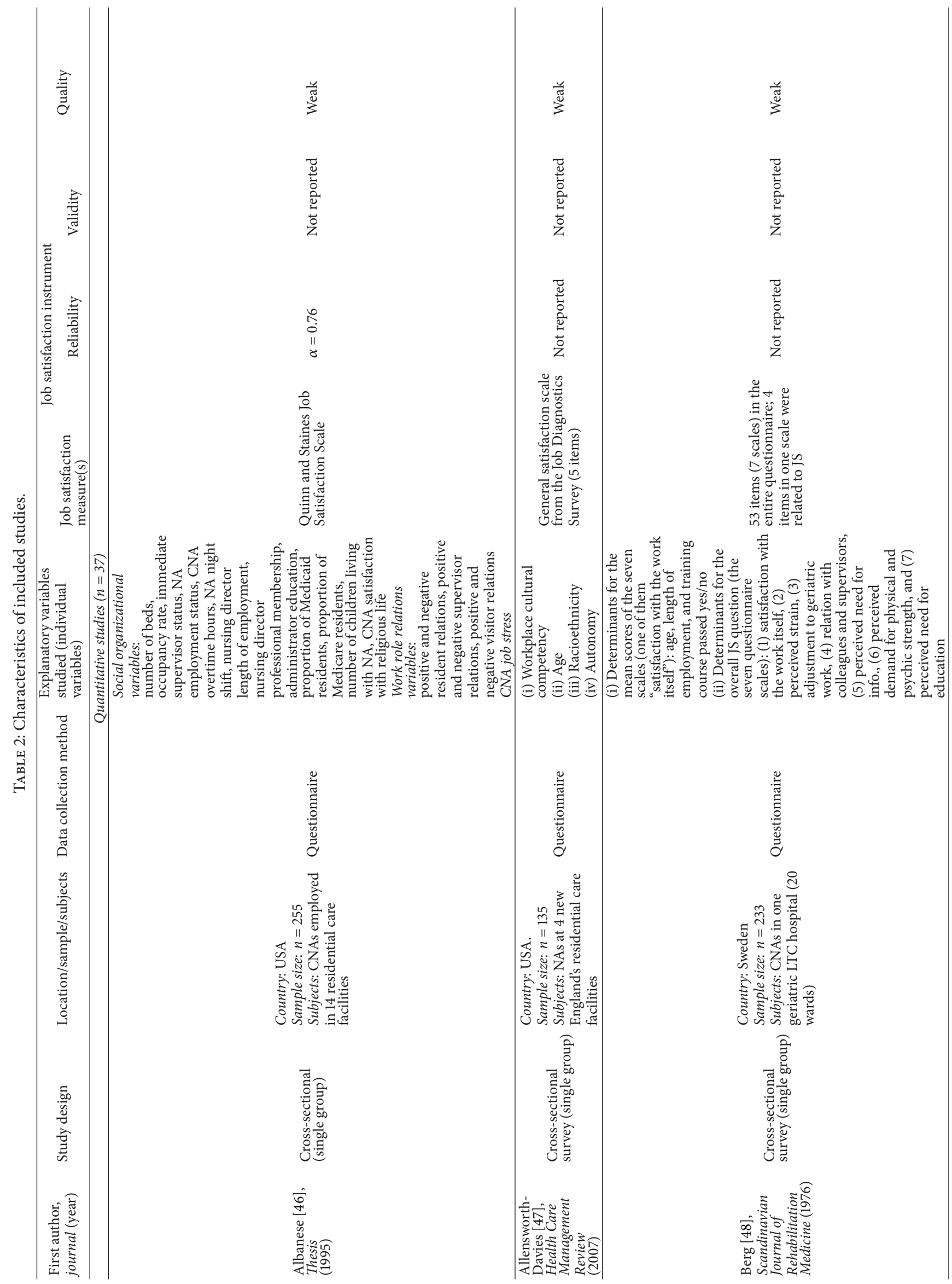




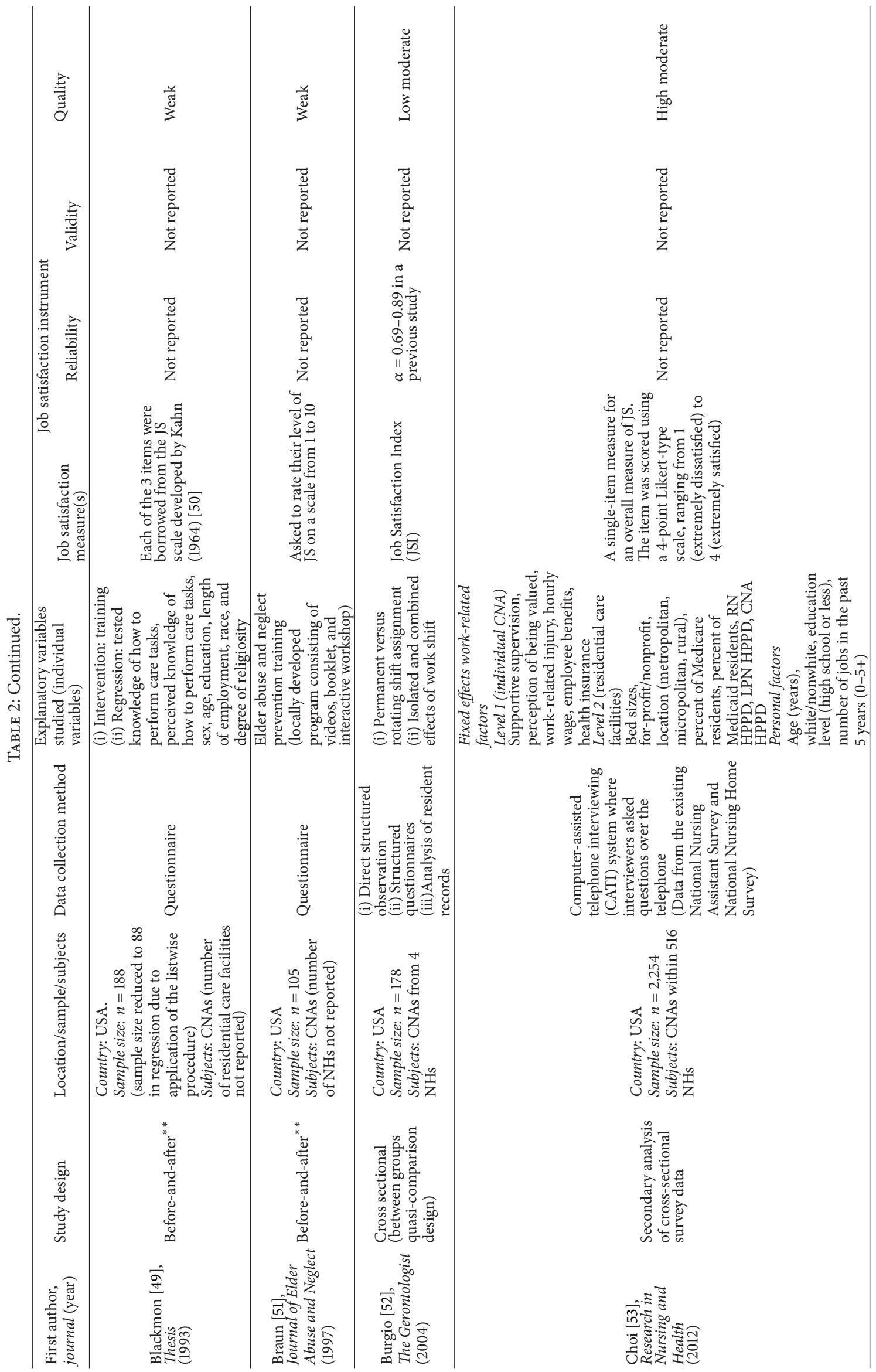




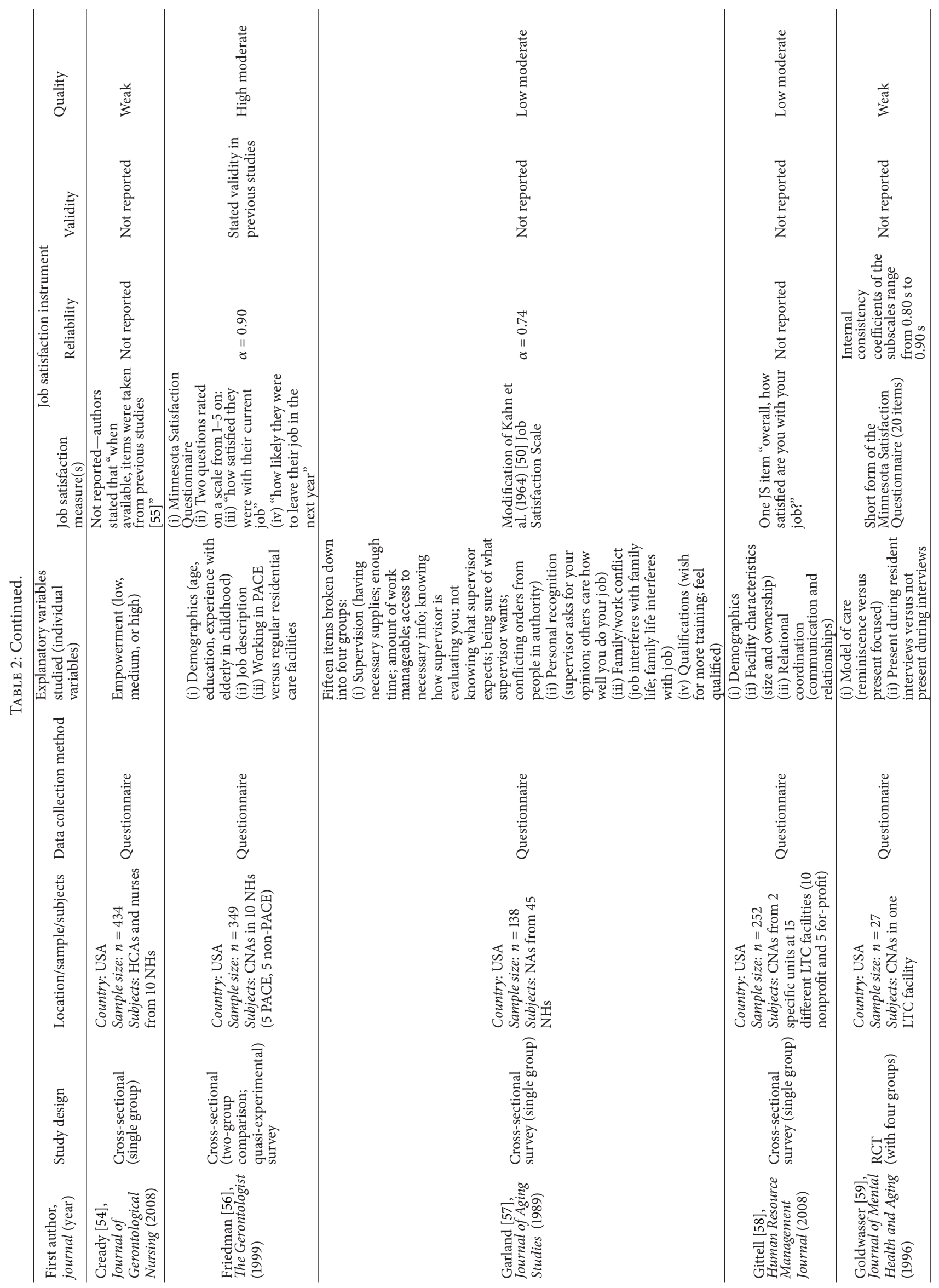




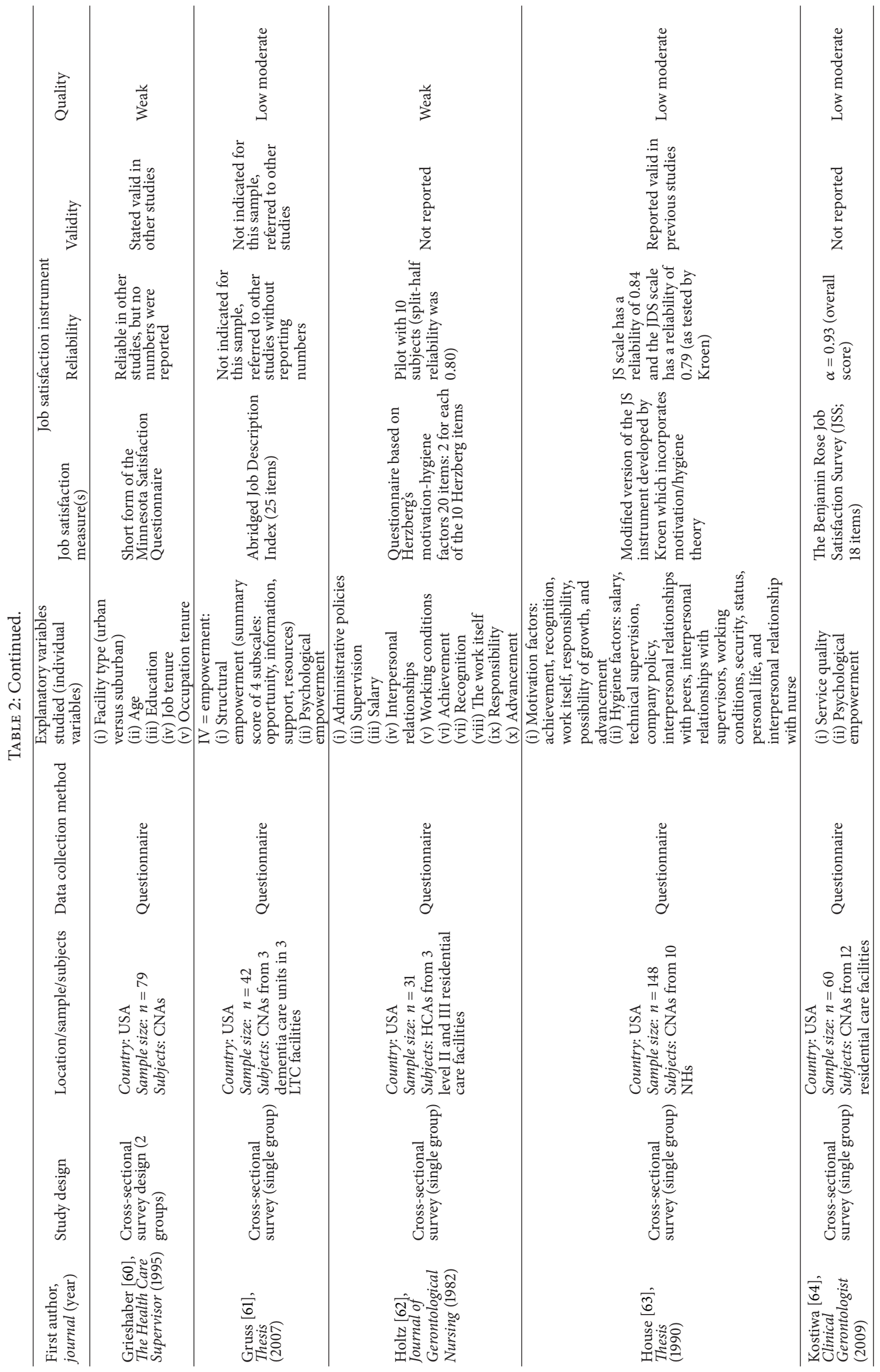




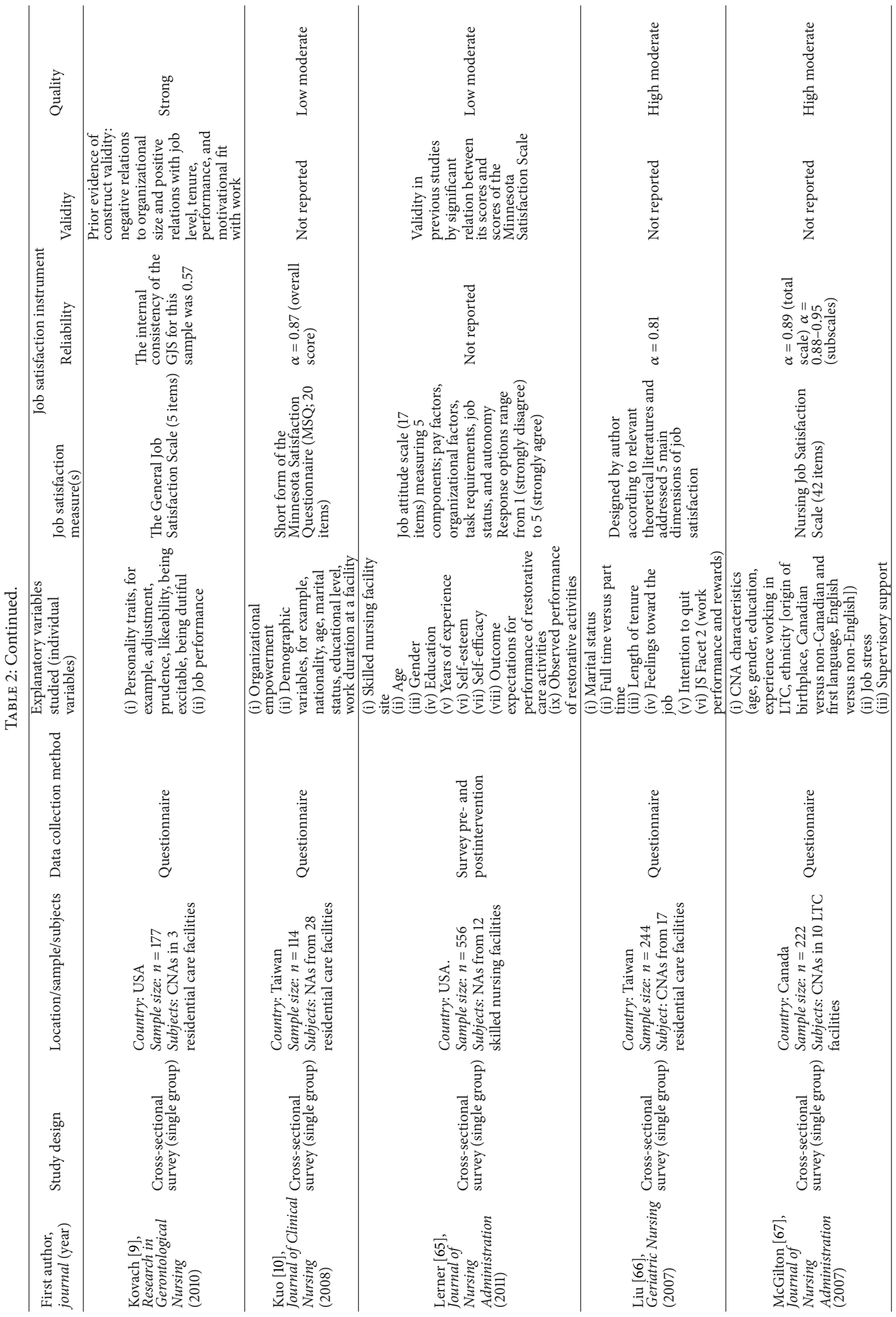




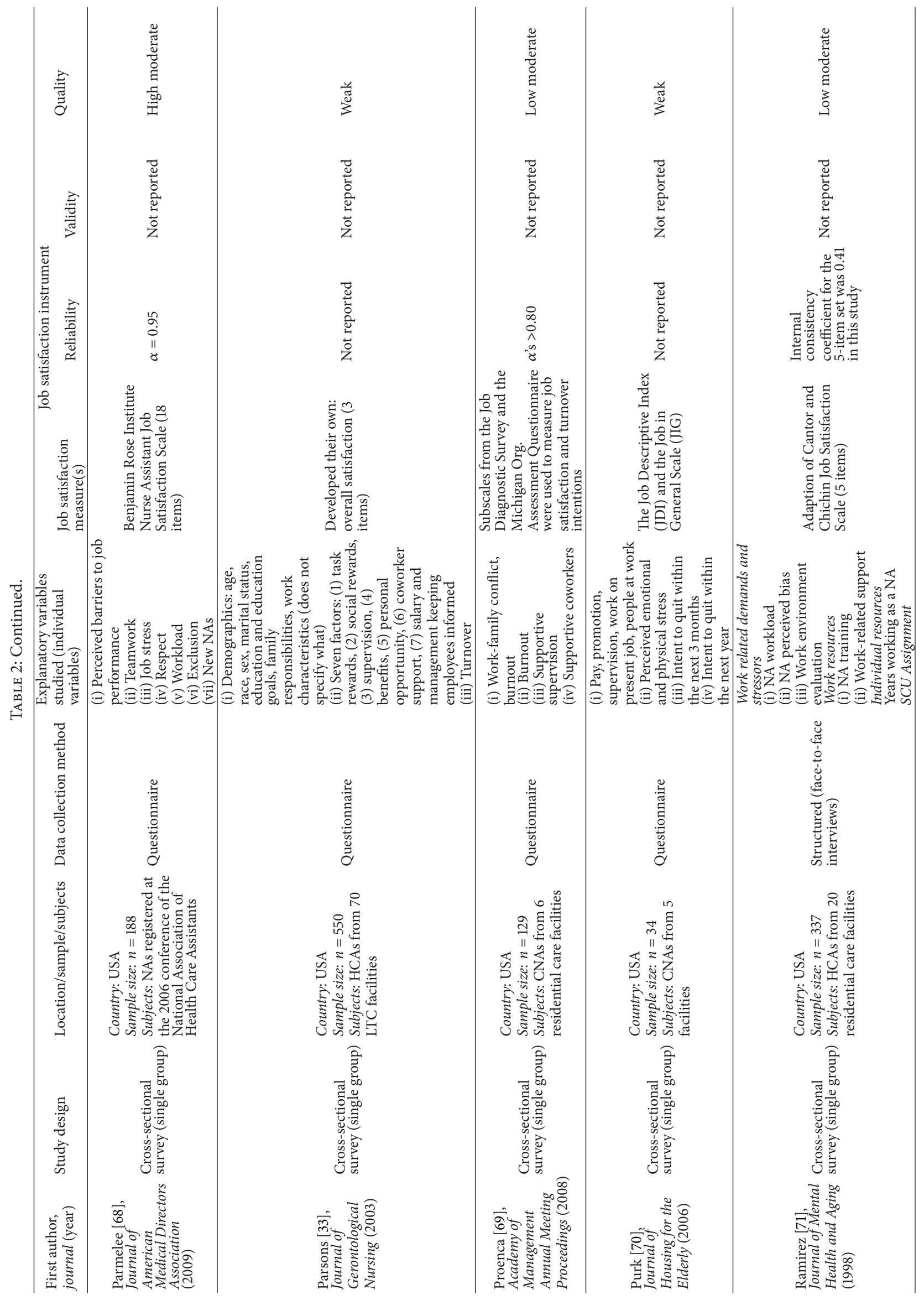




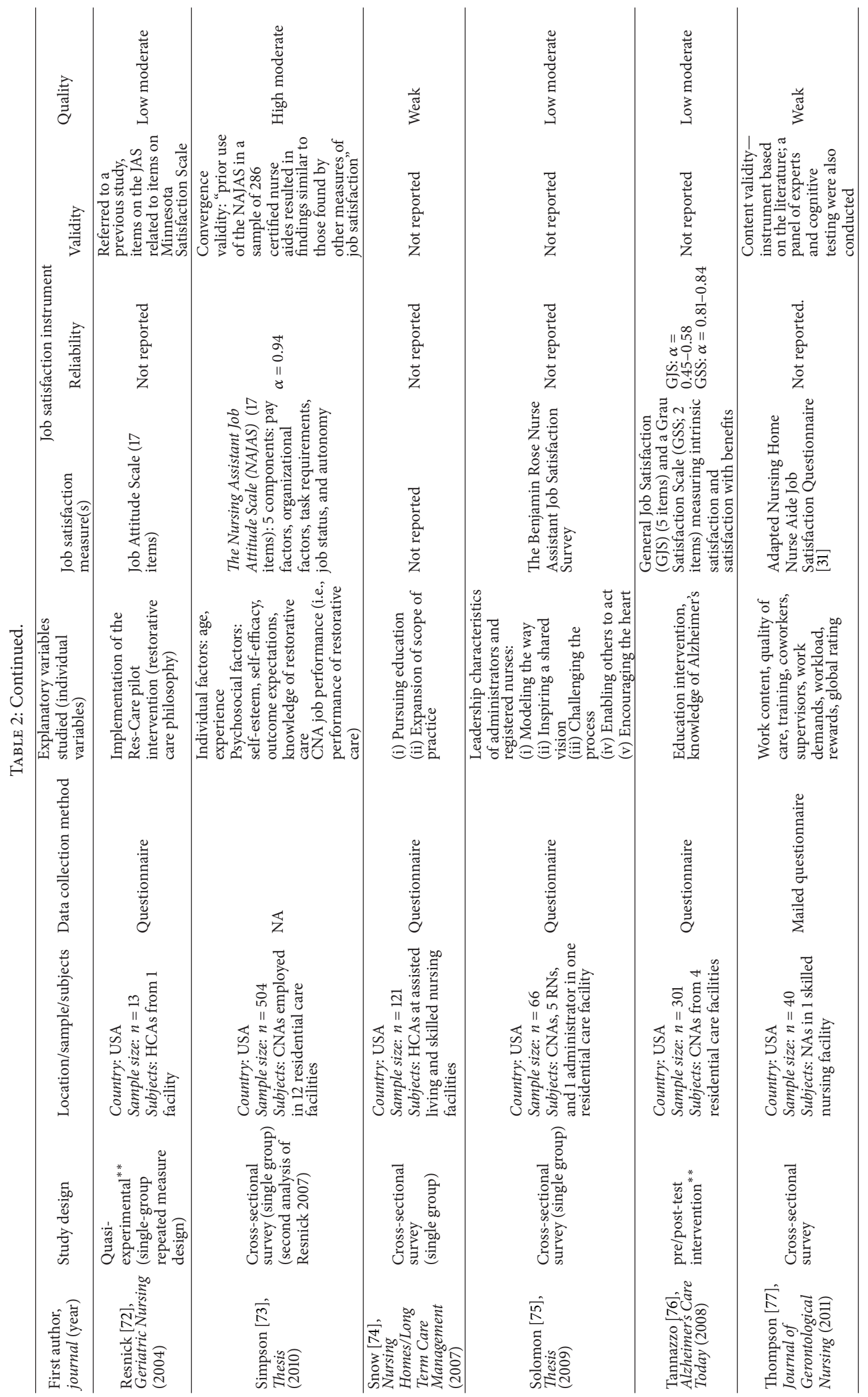




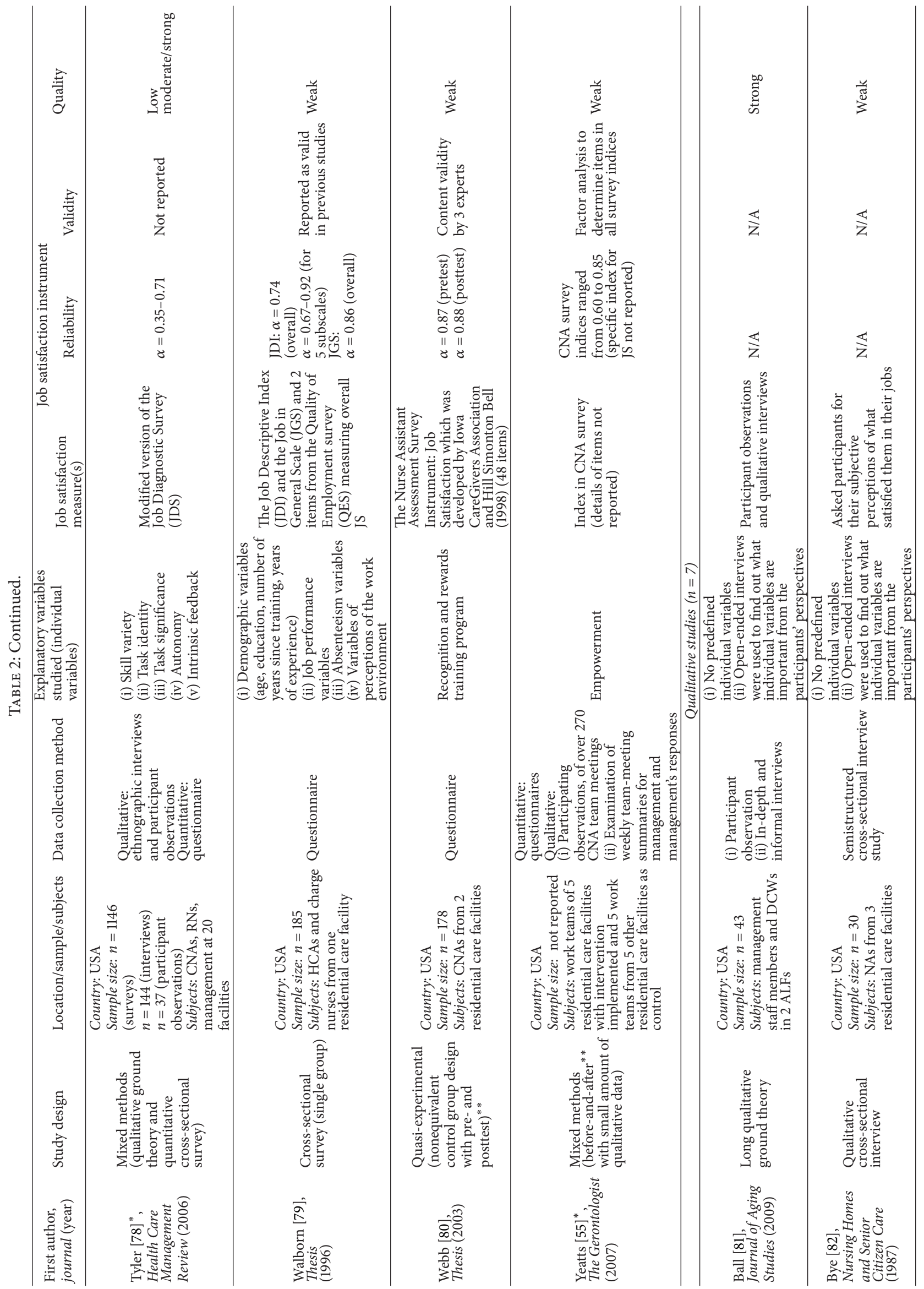




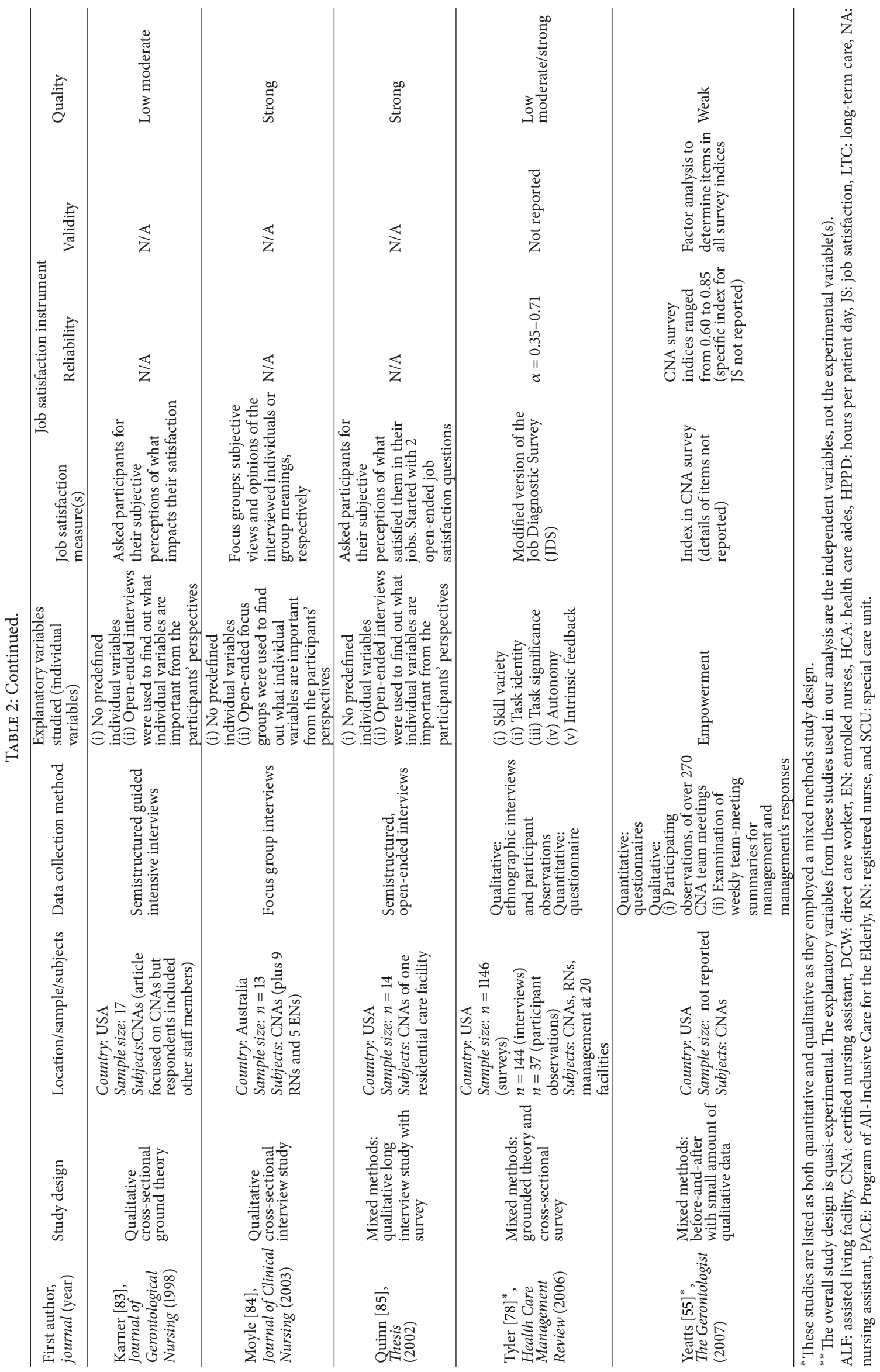


TABLE 3: Individual factors (reported four or more times).

\begin{tabular}{|c|c|c|c|c|c|}
\hline Category & First author & $\begin{array}{l}\text { Significance }(\mathrm{S} \\
=p<.05)\end{array}$ & $\begin{array}{c}\text { Direction } \\
\text { (magnitude) }\end{array}$ & $\begin{array}{l}\text { Methodological } \\
\text { quality }\end{array}$ & Sample size \\
\hline \multicolumn{6}{|c|}{ (1) Sociodemographics ( $n=13$ studies) } \\
\hline \multirow{12}{*}{$\begin{array}{l}\text { Age }(n=12 \\
\text { studies })\end{array}$} & Allensworth-Davies [47] & NS & & Weak & 135 \\
\hline & Blackmon [49] & NS & & Weak & 188 \\
\hline & Choi $[53]$ & NS & & High moderate & 2,254 \\
\hline & Friedman $[56]$ & S & $+(\beta=0.15)$ & High moderate & 349 \\
\hline & Gittell [58] & NS & & Low moderate & 252 \\
\hline & Grieshaber [60] & NS & & Weak & 79 \\
\hline & Kuo $[10]$ & NS & & Low moderate & 114 \\
\hline & Lerner $[65]$ & NS & & Low moderate & 556 \\
\hline & McGilton [67] & NS & & High moderate & 222 \\
\hline & Parsons [33] & NS & & Weak & 550 \\
\hline & Simpson [73] & S & $+(\beta=0.14)$ & High moderate & 504 \\
\hline & Walborn [79] & S & $+(r=0.218)$ & Weak & 185 \\
\hline \multirow{7}{*}{$\begin{array}{l}\text { Ethnicity ( } n=7 \\
\text { studies) }\end{array}$} & Allensworth-Davies [47] & NS & & Weak & 135 \\
\hline & Blackmon [49] & NS & & Weak & 188 \\
\hline & Choi $[53]$ & NS & & High moderate & 2,254 \\
\hline & Kuo [10] & S & $+(\beta=0.32)$ & Low moderate & 114 \\
\hline & McGilton [67] & $\mathrm{S}$ & $-(\beta=-0.28)$ & High moderate & 222 \\
\hline & Parsons [33] & NS & & Weak & 550 \\
\hline & Ramirez [71] & $\begin{array}{l}\mathrm{S} \text { (for } 2 / 3 \\
\text { races) }\end{array}$ & $\begin{array}{c}-(\beta=-0.14 \\
\text { to }-0.20)\end{array}$ & Low moderate & 337 \\
\hline \multirow{6}{*}{$\begin{array}{l}\text { Gender ( } n=6 \\
\text { studies) }\end{array}$} & Blackmon [49] & NS & & Weak & 188 \\
\hline & Gittell [58] & NS & & Low moderate & 252 \\
\hline & Kuo $[10]$ & NS & & Low moderate & 114 \\
\hline & Lerner $[65]$ & NS & & Low moderate & 556 \\
\hline & McGilton [67] & NS & & High moderate & 222 \\
\hline & Parsons [33] & NS & & Weak & 550 \\
\hline \multicolumn{6}{|c|}{ (2) Education ( $n=17$ studies) } \\
\hline \multirow{11}{*}{$\begin{array}{l}\text { Level of } \\
\text { education/years } \\
\text { Education ( } n=10 \\
\text { studies) }\end{array}$} & Blackmon [49] & NS & & Weak & 188 \\
\hline & Choi $[53]$ & NS & & High moderate & 2,254 \\
\hline & Friedman [56] & NS & & High moderate & 349 \\
\hline & Gittell [58] & NS & & Low moderate & 252 \\
\hline & Goldwasser [59] & S & $-{ }^{*}$ & Weak & 27 \\
\hline & Grieshaber [60] & NS (urban) & & Weak & 79 \\
\hline & Grieshaber [60] & S (suburban) & $-(r=-0.51)$ & Weak & 79 \\
\hline & Kuo $[10]$ & NS & & Low moderate & 114 \\
\hline & Lerner [65] & NS & & Low moderate & 556 \\
\hline & Parsons [33] & NS & & Weak & 550 \\
\hline & Walborn [79] & S & $\begin{array}{c}-(r= \\
-0.274)\end{array}$ & Weak & 185 \\
\hline \multirow{8}{*}{$\begin{array}{l}\text { Special training ( } n \\
=8 \text { studies) }\end{array}$} & Blackmon [49] & NS & & Weak & 188 \\
\hline & Braun [51] & S & $+^{*}$ & Weak & 105 \\
\hline & Ramirez [71] & $\mathrm{S}$ & $-(r=-0.13)$ & Low moderate & 337 \\
\hline & Resnick [72] & NS & & Low moderate & 13 \\
\hline & Simpson [73] & NS & & High moderate & 504 \\
\hline & Tannazzo [76] & NS & & Low moderate & 301 \\
\hline & Thompson [77] & NS & & Weak & 40 \\
\hline & Webb $[80]$ & NS & & Weak & 178 \\
\hline
\end{tabular}


TABLE 3: Continued.

\begin{tabular}{|c|c|c|c|c|c|}
\hline Category & First author & $\begin{array}{l}\text { Significance (S } \\
=p<.05)\end{array}$ & $\begin{array}{c}\text { Direction } \\
\text { (magnitude) }\end{array}$ & $\begin{array}{c}\text { Methodological } \\
\text { quality }\end{array}$ & Sample size \\
\hline \multicolumn{6}{|c|}{ (3) Healthcare provider characteristics ( $n=18$ studies) } \\
\hline \multirow{5}{*}{$\begin{array}{l}\text { Empowerment } \\
\text { ( } n=5 \text { studies })\end{array}$} & $\begin{array}{l}\text { Cready [54] } \\
\text { (autonomy in decision making and } \\
\text { perceived meaningful work with a } \\
\text { feeling of competence to do it) }\end{array}$ & S & $+^{*}$ & Weak & 434 \\
\hline & $\begin{array}{l}\text { Gruss [61] } \\
\text { (perceived control and access to power } \\
\text { within the organization) }\end{array}$ & S & $+(r=0.46)$ & Low moderate & 42 \\
\hline & $\begin{array}{l}\text { Kostiwa [64] } \\
\text { (transfer of power to nonmanagement } \\
\text { employees) }\end{array}$ & S & $+(\beta=0.294)$ & Low moderate & 60 \\
\hline & $\begin{array}{l}\text { Kuo [10] } \\
\text { (perceived support, access to } \\
\text { information and resources, } \\
\text { opportunity to learn and grow, good } \\
\text { relationships with staff) }\end{array}$ & S & $+(r=0.366)$ & Low moderate & 114 \\
\hline & $\begin{array}{l}\text { Yeatts [55] } \\
\text { (autonomy in decision making and } \\
\text { perceived meaningful work with } \\
\text { competence to do it) }\end{array}$ & NS & & Weak & Not reported \\
\hline \multirow{5}{*}{$\begin{array}{l}\text { Years of experience } \\
\text { ( } n=5 \text { studies })\end{array}$} & McGilton [67] & NS & & High moderate & 222 \\
\hline & Lerner $[65]$ & S & $+(\beta=0.230)$ & Low moderate & 114 \\
\hline & Ramirez [71] & NS & & Low moderate & 337 \\
\hline & Simpson [73] & NS & & High moderate & 504 \\
\hline & Walborn [79] & $\mathrm{S}$ & $+(r=0.204)$ & Weak & 185 \\
\hline \multirow{4}{*}{$\begin{array}{l}\text { Current position } \\
\text { tenure }(n=3 \\
\text { studies })\end{array}$} & Gittell [58] & NS & & Low moderate & 252 \\
\hline & Grieshaber [60] & NS (urban) & & Weak & 79 \\
\hline & Grieshaber [60] & S (suburban) & $+(r=0.38)$ & Weak & 79 \\
\hline & Liu [66] & S & $-(\beta=-0.14)$ & High moderate & 244 \\
\hline \multirow{4}{*}{$\begin{array}{l}\text { Employment status } \\
\text { (rotating, part } \\
\text { time, full time) ( } n \\
=4 \text { studies) }\end{array}$} & Albanese [46] & NS & & Weak & 255 \\
\hline & Burgio [52] & S & $\begin{array}{c}F(1,173)= \\
6.38\end{array}$ & Low moderate & 178 \\
\hline & Liu [66] & $\mathrm{S}$ & $-(\beta=-0.15)$ & High moderate & 244 \\
\hline & McGilton [67] & NS & & High moderate & 222 \\
\hline \multirow{4}{*}{$\begin{array}{l}\text { Autonomy ( } n=3 \\
\text { studies) }\end{array}$} & $\begin{array}{l}\text { Allensworth-Davies [47] } \\
\text { (definition not reported) }\end{array}$ & $\mathrm{S}$ & $+(\beta=0.23)$ & Weak & 135 \\
\hline & $\begin{array}{l}\text { Friedman [56] (opportunity to use } \\
\text { their own judgment) }\end{array}$ & NS & & High moderate & 349 \\
\hline & $\begin{array}{l}\text { Friedman [56] (opportunity to } \\
\text { organize workload) }\end{array}$ & $S$ & $+(\beta=0.17)$ & High moderate & 349 \\
\hline & $\begin{array}{l}\text { Tyler [78] } \\
\text { (degree to which a job provides } \\
\text { independence and discretion in } \\
\text { scheduling work and determining } \\
\text { ways to carry it out) }\end{array}$ & S & $*$ & $\begin{array}{c}\text { Low } \\
\text { moderate/strong }\end{array}$ & 1146 \\
\hline \multicolumn{6}{|c|}{ (4) Personal life ( $n=4$ studies) } \\
\hline \multirow{4}{*}{$\begin{array}{l}\text { Stress }(n=4 \\
\text { studies) }\end{array}$} & Albanese [46] & $\mathrm{S}$ & $-(r=-0.37)$ & Weak & 255 \\
\hline & McGilton [67] & $\mathrm{S}$ & $-(\beta=-0.19)$ & High moderate & 222 \\
\hline & Parmelee [68] & NS & & High moderate & 188 \\
\hline & Purk [70] & NS & & Weak & 34 \\
\hline
\end{tabular}

*: test statistic value not reported; $r$ : estimate of the Pearson product-moment correlation coefficient; $\beta$ : in multiple regression, a standardized coefficient indicating the relative weight of a predictor variable. 
TABLE 4: Organizational factors (reported four or more times).

\begin{tabular}{|c|c|c|c|c|c|}
\hline Category & First author & $\begin{array}{l}\text { Significance } \\
(\mathrm{S}=p<.05)\end{array}$ & $\begin{array}{c}\text { Direction } \\
\text { (magnitude) }\end{array}$ & $\begin{array}{c}\text { Methodological } \\
\text { quality }\end{array}$ & Sample size \\
\hline \multicolumn{6}{|c|}{ (1) Facility ( $n=3$ studies) } \\
\hline \multirow{4}{*}{$\begin{array}{l}\text { Resources } \\
\text { ( } n=3 \text { studies) }\end{array}$} & Garland [57] & S & $+(r=0.43)$ & Low moderate & 138 \\
\hline & $\begin{array}{c}\text { Kuo [10], } \\
\text { information }\end{array}$ & NS & & Low moderate & 114 \\
\hline & Kuo [10], resources & S & $+(\beta=0.32)$ & Low moderate & 114 \\
\hline & Ramirez [71] & S & $+(\beta=0.24)$ & Low moderate & 337 \\
\hline \multicolumn{6}{|c|}{ (2) Work environment ( $n=13$ studies) } \\
\hline \multirow{6}{*}{$\begin{array}{l}\text { Satisfaction with } \\
\text { salary/benefit } \\
\text { ( } n=4 \text { studies) }\end{array}$} & Choi [53], salary & NS & & High moderate & 2,254 \\
\hline & Choi [53], benefits & S & $\mathrm{OR}=1.14^{* *}$ & High moderate & 2,254 \\
\hline & House [63] & NS & & Low moderate & 148 \\
\hline & Parsons [33], salary & NS & & Weak & 550 \\
\hline & $\begin{array}{c}\text { Parsons [33] } \\
\text { benefits }\end{array}$ & NS & & Weak & 550 \\
\hline & Purk [70] & S & * & Weak & 34 \\
\hline \multirow{4}{*}{$\begin{array}{l}\text { Job performance } \\
\text { ( } n=4 \text { studies })\end{array}$} & Kovach [9] & NS & & Strong & 177 \\
\hline & Liu [66] & S & $+(\beta=0.40)$ & High moderate & 244 \\
\hline & Simpson [73] & NS & & High moderate & 504 \\
\hline & Walborn [79] & NS & & Weak & 185 \\
\hline \multirow{6}{*}{$\begin{array}{l}\text { Support from } \\
\text { coworkers } \\
\text { ( } n=6 \text { studies) }\end{array}$} & Friedman [56] & NS & & High moderate & 349 \\
\hline & Kuo $[10]$ & NS & & Low moderate & 114 \\
\hline & Parmelee [68] & $\mathrm{S}$ & $-(\beta=-0.145)$ & High moderate & 188 \\
\hline & Parsons [33] & S & $+(\beta=0.138)$ & Weak & 550 \\
\hline & Proenca [69] & NS & & Low moderate & 129 \\
\hline & Thompson [77] & S & $*$ & Weak & 40 \\
\hline \multicolumn{6}{|c|}{ (3) Workload ( $n=5$ studies) } \\
\hline \multirow{5}{*}{$\begin{array}{l}\text { Workload } \\
\text { ( } n=5 \text { studies })\end{array}$} & $\begin{array}{c}\text { Berg [48] } \\
\text { (perceived strain) }\end{array}$ & S & $-(r=-0.38)$ & Weak & 233 \\
\hline & Garland [57] & $\mathrm{S}$ & $+(r=0.3)$ & Low moderate & 138 \\
\hline & Parmelee [68] & S & $-(\beta=-0.283)$ & High moderate & 188 \\
\hline & Ramirez [71] & S & $-(\beta=-0.21)$ & Low moderate & 337 \\
\hline & Thompson [77] & S & $*$ & Weak & 40 \\
\hline
\end{tabular}

*: test statistic value not reported; $* *: \chi^{2}$ not reported; $r$ : estimate of the Pearson product-moment correlation coefficient; $\beta$ : in multiple regression, a standardized coefficient indicating the relative weight of a predictor variable.

were most frequently mentioned in both quantitative and qualitative studies; respondents in all 7 qualitative studies discussed 1 or more work environment factors. Of particular significance is that 3 factors not studied quantitatively emerged in the qualitative studies as important to care aide job satisfaction: contact/relationships with residents [82-85], nature of the job (care aide work) $[62,84,85]$, and opportunity for learning and advancement [62, 74, 82].

\section{Discussion}

4.1. Summary of Findings. This systematic review examined the evidence on associations between individual and organizational factors and care aide job satisfaction. The body of evidence provides significant empirical support for the relationship of several factors to an increase in care aides' job satisfaction. Important individual factors identified were empowerment and autonomy. Six individual factors were shown to be not important: age, ethnicity, gender, education level, attending specialized training, and years of experience. Important organizational factors were facility resources and workload. Two organizational factors were found to be not important: care aide satisfaction with salary/benefits and job performance.

4.2. Comparison with the Review on Job Satisfaction among Hospital Registered Nurses. No previous syntheses exist on job satisfaction in care aides or with nursing care providers in residential LTC, but job satisfaction among registered nurses in hospitals was subject to a recent systematic review [20]. Both studies found the individual factors autonomy and empowerment to be important to job satisfaction. However, 
TABLE 5: Individual factor conclusions.

\begin{tabular}{|c|c|c|}
\hline \multicolumn{3}{|c|}{ Sociodemographic } \\
\hline Age & $3 / 12(25 \%)$ reports significant & $\begin{array}{l}\text { No relationship with job } \\
\text { satisfaction }\end{array}$ \\
\hline Ethnicity & $3 / 7(43 \%)$ reports significant & $\begin{array}{l}\text { No relationship with job } \\
\text { satisfaction }\end{array}$ \\
\hline Gender & $0 / 6(0 \%)$ reports significant & $\begin{array}{l}\text { No relationship with job } \\
\text { satisfaction }\end{array}$ \\
\hline \multicolumn{3}{|c|}{ Education } \\
\hline $\begin{array}{l}\text { Level of education/years } \\
\text { Education }\end{array}$ & $3 / 11(27 \%)$ reports significant & $\begin{array}{l}\text { No relationship with job } \\
\text { satisfaction }\end{array}$ \\
\hline Special training & $2 / 8(25 \%)$ reports significant & $\begin{array}{l}\text { No relationship with job } \\
\text { satisfaction }\end{array}$ \\
\hline \multicolumn{3}{|c|}{ Professional characteristics } \\
\hline Empowerment & $4 / 5(80 \%)$ reports significant & $\begin{array}{l}\text { Positive relationship with job } \\
\text { satisfaction }\end{array}$ \\
\hline Years of experience & $2 / 5(40 \%)$ reports significant & $\begin{array}{l}\text { No relationship with job } \\
\text { satisfaction }\end{array}$ \\
\hline Current position & $2 / 4(50 \%)$ reports significant & $\begin{array}{l}\text { Equivocal relationship with job } \\
\text { satisfaction }\end{array}$ \\
\hline Employment status & $2 / 4(50 \%)$ reports significant & $\begin{array}{l}\text { Equivocal relationship with job } \\
\text { satisfaction }\end{array}$ \\
\hline Autonomy & $3 / 4(75 \%)$ reports significant & $\begin{array}{l}\text { Positive relationship with job } \\
\text { satisfaction }\end{array}$ \\
\hline \multicolumn{3}{|c|}{ Personal life } \\
\hline Stress & $2 / 4(50 \%)$ reports significant & $\begin{array}{l}\text { Equivocal relationship with job } \\
\text { satisfaction }\end{array}$ \\
\hline
\end{tabular}

TABLE 6: Organizational factor conclusions.

\begin{tabular}{lcc}
\hline & \multicolumn{1}{c}{ Facility } & \\
\hline Resources & \multicolumn{1}{c}{ W/4 (75\%) reports significant } & Positive relationship with job satisfaction \\
\hline Satisfaction with salary/benefits & $2 / 6(33 \%)$ reports significant & No relationship with job satisfaction \\
Job performance & $1 / 4(25 \%)$ reports significant & No relationship with job satisfaction \\
Support from coworkers & $3 / 6(50 \%)$ reports significant & Equivocal relationship with job satisfaction \\
\hline & & Workload \\
\hline Workload & $5 / 5(100 \%)$ reports significant & Positive relationship with job satisfaction \\
\hline
\end{tabular}

several important differences between our review and the hospital registered nurse review are evident. First, in the hospital registered nurse group, job satisfaction was closely related to working conditions and organizational and environmental factors, namely, job stress, role conflict/ambiguity, role perception/content, organizational commitment, and professional commitment. While we found similar overall categories, we found different factors within these categories to be important to care aide job satisfaction in residential LTC. For example, both nurse job satisfaction and care aide job satisfaction were closely related to working conditions, but care aides noted workloads and availability of facility level resources as important (Table 4) while registered hospital nurses noted team cohesiveness and physical conditions of the unit to be important [20]. Coworker support had a high moderate relationship to hospital registered nurse job satisfaction [20] but was only equivocally related to care aide job satisfaction (Table 4). Second, while age, years of experience, and education level all had significant relationships with job satisfaction in hospital registered nurses [20], these individual factors were not consistently significant to care aide job satisfaction (Table 3 ). Third, stress had a strong relationship with registered nurse job satisfaction [20] but only an equivocal relationship for care aides (Table 3). Each of these discrepancies may reflect true differences between groups (i.e., between registered nurses and care aides) and/or settings (i.e., between hospitals and residential LTC) or may reflect differences in synthesis methods. Lu and colleagues [20] reported all factors displaying statistically significant findings in any study as important to registered nurse job satisfaction. In this synthesis, we applied stringent decision rules. To classify a factor as important to job satisfaction, we required it to be tested 4 or more times and have significant findings in at least $60 \%$ of those studies. Regardless 
TABLE 7: Summary of qualitative findings.

\begin{tabular}{|c|c|c|}
\hline Factor & First author & Details \\
\hline \multicolumn{3}{|r|}{ Individual factors } \\
\hline \multicolumn{3}{|l|}{ Education } \\
\hline Pursuing education & Snow $[74]$ & CNAs reported they would have greater job satisfaction with more education/expanded skills \\
\hline $\begin{array}{l}\text { Pursuing nursing } \\
\text { career }\end{array}$ & Snow $[74]$ & $\begin{array}{l}\text { (i) CNAs pursuing a nursing career reported the highest level of job satisfaction, followed by } \\
\text { CNAs with no plans for further education } \\
\text { (ii) CNAs pursuing education outside of health care reported the lowest levels of job } \\
\text { satisfaction }\end{array}$ \\
\hline \multicolumn{3}{|l|}{ Other } \\
\hline Feeling needed/useful & Bye [82] & 93\% stated feeling needed/useful was the most satisfying aspect of their work \\
\hline \multicolumn{3}{|r|}{ Organizational factors } \\
\hline \multicolumn{3}{|l|}{ Facility: resources } \\
\hline $\begin{array}{l}\text { Equipment and } \\
\text { supplies }\end{array}$ & Quinn [85] & Mainly positive responses, more resources linking to higher job satisfaction \\
\hline \multicolumn{3}{|l|}{ Facility: other } \\
\hline Workplace flexibility & Moyle [84] & Related to job satisfaction \\
\hline $\begin{array}{l}\text { Working on skilled } \\
\text { units }\end{array}$ & Bye [82] & Some enjoyed challenge of working on skilled units \\
\hline Facility & Bye [82] & Some were happy in their current facility and would not like to go to another facility \\
\hline Pay satisfaction & Quinn [85] & Typical responses positive in relation to job satisfaction \\
\hline Benefits satisfaction & Quinn [85] & Many variant responses positive/negative re job satisfaction \\
\hline $\begin{array}{l}\text { Facility's response to } \\
\text { needs and concerns }\end{array}$ & Quinn [85] & Many variant responses positive/negative re job satisfaction \\
\hline $\begin{array}{l}\text { People in } \\
\text { management }\end{array}$ & Quinn [85] & Many variant responses positive/negative re job satisfaction \\
\hline Admin support & Karner [83] & $\begin{array}{l}\text { Contributing to increased job satisfaction-appropriate and kind administrative support; } \\
\text { respectful of aides' knowledge }\end{array}$ \\
\hline \multicolumn{3}{|l|}{ Work environment } \\
\hline $\begin{array}{l}\text { Working with } \\
\text { unskilled or } \\
\text { inappropriately } \\
\text { trained staff }\end{array}$ & Moyle [84] & Related to job dissatisfaction \\
\hline \multirow{3}{*}{$\begin{array}{l}\text { Working conditions } \\
\text { Organizational } \\
\text { structure }\end{array}$} & Holtz [62] & $68 \%$ of aides said that they were extremely or very important to their job satisfaction \\
\hline & Karner [83] & $\begin{array}{l}\text { Contributing to increased job satisfaction-fair and consistent organizational structures; } \\
\text { hands-on training and adequate staff }\end{array}$ \\
\hline & Holtz [62] & $77 \%$ of aides said that it was extremely or very important \\
\hline Recognition/respect & Quinn [85] & $\begin{array}{l}\text { Many variant responses-some say recognition for work is important to job satisfaction and } \\
\text { others lead to job dissatisfaction }\end{array}$ \\
\hline \multirow{7}{*}{ Residents } & Quinn [85] & Typical response negative for quantity of recognition leading to job satisfaction \\
\hline & Walborn [79] & Nurse aides would like more respect, for example, from family members \\
\hline & Bye $[82]$ & Most identified their interaction with residents as the most satisfying aspect of their job \\
\hline & Quinn [85] & Many variant responses, typical response positive in relation to job satisfaction \\
\hline & Moyle [84] & $\begin{array}{l}\text { (i) Related to job satisfaction } \\
\text { (ii) Contact with residents promotes enjoyment and job satisfaction } \\
\text { (iii) Job satisfaction comes from resident: interactions and appreciation }\end{array}$ \\
\hline & Walborn [79] & Interacting with residents was a satisfying aspect of the job \\
\hline & Karner [83] & Relation with residents was a satisfying aspect of the job \\
\hline $\begin{array}{l}\text { Family member } \\
\text { participation in } \\
\text { resident care }\end{array}$ & Karner [83] & Contributing to increased job satisfaction \\
\hline \multirow{3}{*}{$\begin{array}{l}\text { Interpersonal } \\
\text { relationships }\end{array}$} & Quinn [85] & Typical response positive in relation to job satisfaction \\
\hline & Holtz [62] & $100 \%$ of aides said that interpersonal relationships were important or extremely important \\
\hline & Bye [82] & $53 \%$ said these were 2 nd and 3 rd greatest satisfiers \\
\hline
\end{tabular}


TABLe 7: Continued.

\begin{tabular}{|c|c|c|}
\hline Factor & First author & Details \\
\hline \multirow{3}{*}{$\begin{array}{l}\text { Support from } \\
\text { coworkers }\end{array}$} & Moyle [84] & $\begin{array}{l}\text { (i) Good teamwork increases job satisfaction } \\
\text { (ii) Job dissatisfaction occurs when staff members are intolerant/upset }\end{array}$ \\
\hline & Karner [83] & Contributing to increased job satisfaction \\
\hline & Quinn [85] & Typical response positive in relation to job satisfaction \\
\hline $\begin{array}{l}\text { Tensions within role } \\
\text { expectations }\end{array}$ & Moyle [84] & Related to job dissatisfaction \\
\hline Absenteeism & Quinn [85] & Typical responses negative in relation to job satisfaction \\
\hline $\begin{array}{l}\text { Environment } \\
\text { (homelike) }\end{array}$ & Karner [83] & Contributing to increased job satisfaction \\
\hline Building design & Quinn [85] & Many variant responses positive in relation to job satisfaction \\
\hline Positive feedback & Tyler [78] & $\begin{array}{l}\text { Positive feedback often comes from residents and this type of feedback is more important than } \\
\text { feedback received from supervisors }\end{array}$ \\
\hline $\begin{array}{l}\text { Communication- } \\
\text { valued } \\
\text { input }\end{array}$ & Quinn [85] & Many variant responses negative in relation to job satisfaction \\
\hline Respect & Walborn [79] & Nurse aides would like more respect, for example, from family members \\
\hline \multicolumn{3}{|l|}{ Supervision } \\
\hline Sunervision & Holtz [62] & $90 \%$ of aides said that it was extremely or very important \\
\hline & Walborn [79] & Nursing assistants would like to be listened to by charge nurses/managers \\
\hline \multicolumn{3}{|r|}{ 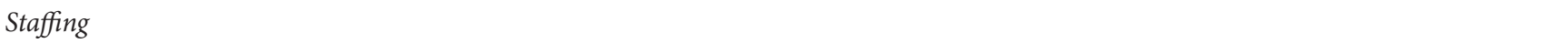 } \\
\hline $\begin{array}{l}\text { Number of staff and } \\
\text { workloads }\end{array}$ & Quinn [85] & Mainly positive responses with respect to more staff linking to higher job satisfaction \\
\hline Staffing levels & Moyle [84] & $\begin{array}{l}\text { (i) Job satisfaction decreases when tasks and time constraints prevent the opportunity to relate } \\
\text { to residents and increases likelihood of error } \\
\text { (ii) Dissatisfied with anything that took them away from resident care }\end{array}$ \\
\hline $\begin{array}{l}\text { Increasing need to be } \\
\text { available for overtime }\end{array}$ & Moyle [84] & $\begin{array}{l}\text { (i) Related to job dissatisfaction } \\
\text { (ii) Overtime created both job satisfaction and dissatisfaction }\end{array}$ \\
\hline \multicolumn{3}{|l|}{$\begin{array}{l}\text { Other: opportunity for } \\
\text { learning and } \\
\text { advancement }\end{array}$} \\
\hline $\begin{array}{l}\text { Learning and growing } \\
\text { on the job }\end{array}$ & Bye [82] & $17 \%$ said this was 2 nd and 3 rd greatest satisfiers \\
\hline $\begin{array}{l}\text { Expansion of scope of } \\
\text { practice }\end{array}$ & Snow $[74]$ & $\begin{array}{l}\text { Overall } 92 \% \text { of the certified nursing assistants believed that expansion of their scope of practice } \\
\text { would increase their job satisfaction }\end{array}$ \\
\hline Advancement & Holtz $[62]$ & $48 \%$ of aides said that it was extremely or very important \\
\hline \multicolumn{3}{|l|}{$\begin{array}{l}\text { Other: nature of the } \\
\text { job }\end{array}$} \\
\hline \multirow{3}{*}{ Work itself } & Holtz [62] & $84 \%$ of aides said that it was extremely or very important \\
\hline & Quinn [85] & Many variant responses in relation to job satisfaction \\
\hline & Moyle $[84]$ & $\begin{array}{l}\text { (i) Laborious tasks (such as documentation) related to job dissatisfaction } \\
\text { (ii) Job dissatisfaction occurs when tensions are not recognized in the workplace: managerial } \\
\text { staff not listening to concerns }\end{array}$ \\
\hline
\end{tabular}

of the reason(s) for differences between the two reviews, these differences highlight the importance of conducting systematic reviews in LTC and with nursing care provider groups other than registered nurses. These findings also importantly suggest that different strategies may be needed to improve care aide job satisfaction in residential LTC facilities compared to hospital nurses.

4.3. Methodological Implications for Future Research. Systematic reviews typically identify problems with internal validity of research under investigation. Future studies on factors related to care aide job satisfaction need to emphasize methodological quality, to reduce bias and increase confidence in this growing body of knowledge. Researchers will then be able to design better-informed interventions to improve care aide job satisfaction, recruitment, and retention of this vital staffing group.

Two important methodological limitations of the studies conducted to date included in this review are methodological quality and statistical rigor. Few studies included in this review were of high moderate or strong methodological quality, illustrating a clear need for well-designed, robust studies in the area. Studies also varied in statistical rigor, although we observed a promising trend in recent studies to more robust analyses (multivariate regression over bivariate and univariate statistics). Given the heterogeneity among 
studies, however, we could only draw conclusions on which factors are associated with job satisfaction and not on which factors predict job satisfaction. Future research should look more closely at prediction; only 14 (38\%) of our 37 included quantitative studies reported prediction (multivariate regression).

4.4. Limitations of This Review. While we used rigorous methods in this review, there are limitations. First, we did not search all grey literature databases; therefore, this review may not include all relevant work. Second, we did not attempt to clarify unclear study details by contacting the study authors; nonreported aspects of methods may have lowered scores in our quality assessment. Third, we used vote counting to synthesize quantitative data. Vote counting does not account for effect sizes (it gives equal weight to all associations irrespective of magnitude) or precision of estimates (it gives equal weight to comparisons irrespective of sample size). To lessen these problems we reported the number of comparisons showing statistically significant effects (regardless of direction) and the magnitude of effect for significant findings [92]. Fourth, there is a small possibility of a culture effect given the fact that different countries may experience job satisfaction differently and also have different determinants to job satisfaction. This effect should however be minimal given the fact that the vast majority of studies identified are from the USA ( $n=37$ of 42). Finally, our criteria for reaching a conclusion on the factors important to job satisfaction were stringent and while we considered overall methodological quality of the included studies in determining these conclusions, we did not take into account specific individual methodological strengths and weaknesses of each study in determining which factors were important overall to care aide job satisfaction.

\section{Conclusions}

We identified several factors as important to care aide job satisfaction. Individual factors were empowerment and autonomy; organizational factors were facility resources and workload. Equally important, several factors were shown to be not important: age, ethnicity, gender, education level, attending specialized training, years of experience, satisfaction with salary/benefits, and job performance. Factors identified as important hold promise as targets of care aide job satisfaction interventions. However, methodological problems inherent in many studies suggest that additional research using more robust study designs and multivariate assessment methods is required. Future research might also usefully test the association between care aide job satisfaction and the factors identified in qualitative studies included in this review: contact/relationships with residents, nature of the job (care aide work), and opportunity for learning and advancement.

\section{List of Abbreviations}

ARD: Age-related dementia

LTC: Long-term care

$\mathrm{RN}$ : Registered nurse
LPN: Licensed practical nurse

RPN: Registered practical nurse

NA: Nursing assistant/aide

CNA: Certified nurse aide.

\section{Disclosure}

Janet E. Squires holds a University Research Chair in Health Evidence Implementation and a Canadian Institutes for Health Research (CIHR) New Investigator Award. Carole A. Estabrooks holds a CIHR Canada Research Chair in Knowledge Translation. Matthias Hoben holds an Alberta Innovates-Health Solutions (AIHS) Postdoctoral Fellowship.

\section{Conflict of Interests}

The authors declare that they have no competing interests.

\section{Authors' Contribution}

Janet E. Squires and Carole A. Estabrooks conceptualized the study. Janet E. Squires and Heather L. Carleton undertook the articles selection. Janet E. Squires, Matthias Hoben, Stefanie Linklater, Heather L. Carleton, and Nicole Graham undertook data extraction and quality assessment. Janet E. Squires, Stefanie Linklater, and Heather L. Carleton undertook data synthesis. Janet E. Squires drafted the paper. All authors provided critical commentary on the paper and approved the final version.

\section{References}

[1] United Nations Population Division, World Population Ageing 1950-2050, United Nations Population Division, 2002.

[2] Canadian Study of Health and Aging Working Group, "Canadian study of health and aging: study methods and prevalence of dementia," Canadian Medical Association Journal, vol. 150, no. 6, pp. 899-913, 1994.

[3] CIHR, Turning the Tide: A Strategy for International Leadership in the Prevention and Early Treatment of Alzheimer's Disease and Related Dementias, Canadian Institutes of Health Research, Ottawa, Canada, 2010.

[4] Alzheimer's Association, "2013 Alzheimer's disease facts and figures," Alzheimer's \& Dementia, vol. 9, no. 2, pp. 208-245, 2013.

[5] World Health Organization, Alzheimer's Disease International. Dementia: A Public Health Priority, WHO Press, Geneva, Switzerland, 2012.

[6] J. E. Gaugler, S. Duval, K. A. Anderson, and R. L. Kane, "Predicting nursing home admission in the US: a meta-analysis," BMC Geriatrics, vol. 7, article 13, 2007.

[7] H. Trottier, L. Martel, C. Houle, J. M. Berthelot, and J. Légaré, "Living at home or in an institution: what makes the difference for seniors?” Health Reports, vol. 11, no. 4, pp. 49-61, 2000.

[8] G. Van Rensbergen and T. Nawrot, "Medical conditions of nursing home admissions," BMC Geriatrics, vol. 10, article 46, 2010.

[9] C. R. Kovach, M. R. Simpson, A. B. Reitmaier, A. Johnson, and S. T. Kelber, "Do personality traits predict work outcomes of certified nursing assistants?" Research in Gerontological Nursing, vol. 3, no. 4, pp. 253-261, 2010. 
[10] H.-T. Kuo, T. J.-C. Yin, and I.-C. Li, "Relationship between organizational empowerment and job satisfaction perceived by nursing assistants at long-term care facilities," Journal of Clinical Nursing, vol. 17, no. 22, pp. 3059-3066, 2008.

[11] F. Dunn, Report of the Auditor General on Seniors Care and Programs, Auditor General, Edmonton, Canada, 2005.

[12] N. Janes, S. Sidani, C. Cott, and S. Rappolt, "Figuring it out in the moment: a theory of unregulated care providers' knowledge utilization in dementia care settings," Worldviews on EvidenceBased Nursing, vol. 5, no. 1, pp. 13-24, 2008.

[13] P. C. Kontos, K.-L. Miller, G. J. Mitchell, and C. A. Cott, "Dementia care at the intersection of regulation and reflexivity: a critical realist perspective," Journals of Gerontology: Series B: Psychological Sciences and Social Sciences, vol. 66, no. 1, pp. 119128, 2011.

[14] Institute of Medicine (IOM), Retooling for an Aging America: Building the Health Care Workforce, The National Academies Press, Washington, DC, USA, 2008.

[15] B. J. Bowers, S. Esmond, and N. Jacobson, "Turnover reinterpreted: CNAs talk about why they leave," Journal of Gerontological Nursing, vol. 29, no. 3, pp. 36-43, 2003.

[16] C. A. Estabrooks, J. E. Squires, H. L. Carleton, G. G. Cummings, and P. G. Norton, "Who is looking after Mom and Dad? Unregulated workers in Canadian long-term care homes," Canadian Journal on Aging, vol. 34, no. 1, pp. 47-59, 2015.

[17] OECD, A Good Life in Old Age, European Commission, 2013.

[18] M. Kingma, "Nurses on the move: a global overview," Health Services Research, vol. 42, no. 3, pp. 1281-1298, 2007.

[19] L. H. Aiken, S. P. Clarke, D. M. Sloane et al., "Nurses' reports on hospital care in five countries," Health Affairs, vol. 20, no. 3, pp. 43-53, 2001.

[20] H. Lu, K. L. Barriball, X. Zhang, and A. E. While, "Job satisfaction among hospital nurses revisited: a systematic review," International Journal of Nursing Studies, vol. 49, no. 8, pp. 10171038, 2012.

[21] G. A. Zangaro and K. L. Soeken, "A meta-analysis of studies of nurses' job satisfaction," Research in Nursing and Health, vol. 30, no. 4, pp. 445-458, 2007.

[22] J. Cohen-Mansfield, "Turnover among nursing home staff. A review," Nursing Management, vol. 28, no. 5, pp. 59-64, 1997.

[23] C. Donoghue, "Nursing home staff turnover and retention: an analysis of national level data," Journal of Applied Gerontology, vol. 29, no. 1, pp. 89-106, 2010.

[24] S. J. Cavanagh and D. A. Coffin, "Staff turnover among hospital nurses," Journal of Advanced Nursing, vol. 17, no. 11, pp. 13691376, 1992.

[25] M. A. Blegen, "Nurses' job satisfaction: a meta-analysis of related variables," Nursing Research, vol. 42, no. 1, pp. 36-41, 1993.

[26] D. M. Irvine and M. G. Evans, "Job satisfaction and turnover among nurses: integrating research findings across studies," Nursing Research, vol. 44, no. 4, pp. 246-253, 1995.

[27] J. L. Price, "Reflections on the determinants of voluntary turnover," International Journal of Manpower, vol. 22, no. 7, pp. 600-624, 2001.

[28] E. A. Locke, "What is job satisfaction?" Organizational Behavior and Human Performance, vol. 4, no. 4, pp. 309-336, 1969.

[29] E. A. Locke, "The nature and causes of job satisfaction," in Handbook of Industrial and Organizational Psychology, M. D. Dunnette, Ed., Rand-McNally, Chigaco, Ill, USA, 1976.
[30] M. Rose, "Good deal, bad deal? Job satisfaction in occupations," Work, Employment and Society, vol. 17, no. 3, pp. 503-530, 2003.

[31] N. G. Castle, H. Degenholtz, and J. Rosen, "Determinants of staff job satisfaction of caregivers in two nursing homes in Pennsylvania," BMC Health Services Research, vol. 6, article 60, 2006.

[32] P. E. Spector, Job Satisfaction: Application, Assessment, Causes, and Consequences, Sage, Thousand Oaks, Calif, USA, 1997.

[33] S. K. Parsons, W. P. Simmons, K. Penn, and M. Furlough, "Determinants of satisfaction and turnover among nursing assistants. The results of a statewide survey," Journal of Gerontological Nursing, vol. 29, no. 3, pp. 51-58, 2003.

[34] S.-C. Chou, D. P. Boldy, and A. H. Lee, "Measuring job satisfaction in residential aged care," International Journal for Quality in Health Care, vol. 14, no. 1, pp. 49-54, 2002.

[35] L. Pekkarinen, T. Sinervo, M.-L. Perälä, and M. Elovainio, "Work stressors and the quality of life in long-term care units," Gerontologist, vol. 44, no. 5, pp. 633-643, 2004.

[36] S. C. Eaton, "Beyond 'unloving care': linking human resource management and patient care quality in nursing homes," International Journal of Human Resource Management, vol. 11, no. 3, pp. 591-616, 2000.

[37] D. C. Grabowski and S. L. Mitchell, "Family oversight and the quality of nursing home care for residents with advanced dementia," Medical Care, vol. 47, no. 5, pp. 568-574, 2009.

[38] OCED, The OCED Health Project: Long-Term Care for Older People, OECD Publishing, Paris, France, 2005.

[39] National Advisory Council on Aging, NACA Demands Improvement to Canada's Long Term Care Institutions: Press Release, National Advisory Council on Aging, Ottawa, Canada, 2005.

[40] W. R. Shadish, T. D. Cook, and D. T. Campbell, Experimental and Quasi-Experimental Designs for Generalized Causal Inference, Houghton-Mifflin, Boston, Mass, USA, 2002.

[41] Definition of Nurse Assistant, 2012, http://www.MedicineNet .com.

[42] C. A. Estabrooks, J. A. Floyd, S. Scott-Findlay, K. A. O’Leary, and M. Gushta, "Individual determinants of research utilization: a systematic review," Journal of Advanced Nursing, vol. 43, no. 5, pp. 506-520, 2003.

[43] C. A. Estabrooks, G. G. Cummings, S. A. Olivo, J. E. Squires, C. Giblin, and N. Simpson, "Effects of shift length on quality of patient care and health provider outcomes: systematic review," Quality and Safety in Health Care, vol. 18, no. 3, pp. 181-188, 2009.

[44] J. E. Squires, A. M. Hutchinson, A.-M. Boström, H. M. O'Rourke, S. J. Cobban, and C. A. Estabrooks, "To what extent do nurses use research in clinical practice? A systematic review," Implementation Science, vol. 6, article 21, 2011.

[45] J. E. Squires, C. A. Estabrooks, P. Gustavsson, and L. Wallin, "Individual determinants of research utilization by nurses: a systematic review update," Implementation Science, vol. 6, no. 1 , article 1, 2011.

[46] T. H. Albanese, The Impact of Work Role Relationships on Nursing Assistants' Job Stress and Well-Being, University of Akron, 1995.

[47] D. Allensworth-Davies, J. Leigh, K. Pukstas et al., "Country of origin and racio-ethnicity: are there differences in perceived organizational cultural competency and job satisfaction among nursing assistants in long-term care?" Health Care Management Review, vol. 32, no. 4, pp. 321-329, 2007. 
[48] S. Berg, L. Dahl, O. Dehlin, and B. Hedenrud, "Psychological perception of nursing aides' work. An attitude study in a geriatric hospital," Scandinavian Journal of Rehabilitation Medicine, vol. 8, no. 2, pp. 79-84, 1976.

[49] D. J. Blackmon, Nurse Assistants in Nursing Homes: The Impact of Training on Attitudes, Knowledge, and Job Satisfaction, University of Akron, Akron, Ohio, USA, 1993.

[50] R. Kahn, D. Wolfe, R. Quinn, D. Snoek, and R. Rosenthal, Organizational Stress: Studies in Role Conflict and Ambiguity, Wiley, New York, NY, USA, 1964.

[51] K. L. Braun, K. M. Suzuki, C. E. Cusick, and K. Howard-Carhart, "Developing and testing training materials on elder abuse and neglect for nurse aides," Journal of Elder Abuse \& Neglect, vol. 9, no. 1, pp. 1-15, 1997.

[52] L. D. Burgio, S. E. Fisher, J. K. Fairchild, K. Scilley, and J. M. Hardin, "Quality of care in the nursing home: effects of staff assignment and work shift," Gerontologist, vol. 44, no. 3, pp. 368-377, 2004.

[53] J. Choi and M. Johantgen, "The importance of supervision in retention of CNAs," Research in Nursing and Health, vol. 35, no. 2, pp. 187-199, 2012.

[54] C. M. Cready, D. E. Yeatts, M. M. Gosdin, and H. F. Potts, "CNA empowerment: effects on job performance and work attitudes," Journal of Gerontological Nursing, vol. 34, no. 3, pp. 26-35, 2008.

[55] D. E. Yeatts and C. M. Cready, "Consequences of empowered CNA teams in nursing home settings: a longitudinal assessment," Gerontologist, vol. 47, no. 3, pp. 323-339, 2007.

[56] S. M. Friedman, C. Daub, K. Cresci, and R. Keyser, "A comparison of job satisfaction among nursing assistants in nursing homes and the program of all-inclusive care for the elderly (PACE)," Gerontologist, vol. 39, no. 4, pp. 434-439, 1999.

[57] T. N. Garland, N. Oyabu, and G. A. Gipson, "Job satisfaction among nurse assistants employed in nursing homes: an analysis of selected job characteristics," Journal of Aging Studies, vol. 3, no. 4, pp. 369-383, 1989.

[58] J. H. Gittell, D. Weinberg, S. Pfefferle, and C. Bishop, "Impact of relational coordination on job satisfaction and quality outcomes: a study of nursing homes," Human Resource Management Journal, vol. 18, no. 2, pp. 154-170, 2008.

[59] A. N. Goldwasser and S. M. Auerbach, "Audience-based reminiscence therapy intervention: effects on the morale and attitudes of nursing home residents and staff," Journal of Mental Health and Aging, vol. 2, pp. 101-114, 1996.

[60] L. D. Grieshaber, P. Parker, and J. Deering, "Job satisfaction of nursing assistans in long-term care," The Health Care Supervisor, vol. 13, no. 4, pp. 18-28, 1995.

[61] V. A. Gruss, Relation of Work Empowerment to Job Satisfaction and Absenteeism among Dementia-Care Certified Nursing Assistants in Long-Term Care Facilities, Rush University, 2007.

[62] G. A. Holtz, "Nurses' aides in nursing homes: why are they satisfied?" Journal of gerontological nursing, vol. 8, no. 5, pp. 265271, 1982.

[63] M. D. House, Factors that relate to job satisfaction among nurse aides in long-term care facilities [Ph.D. thesis], Colorado State University, 1990.

[64] I. M. Kostiwa and S. Meeks, "The relation between psychological empowerment, service quality, and job satisfaction among certified nursing assistants," Clinical Gerontologist, vol. 32, no. 3, pp. 276-292, 2009.

[65] N. Lerner, B. Resnick, E. Galik, and L. Flynn, "Job satisfaction of nursing assistants," Journal of Nursing Administration, vol. 41, no. 11, pp. 473-478, 2011.
[66] L.-F. Liu, "Job satisfaction of certified nursing assistants and its influence on the general satisfaction of nursin home residents: an exploratory study in southern Taiwan," Geriatric Nursing, vol. 28, no. 1, pp. 54-62, 2007.

[67] K. S. McGilton, L. McGillis Hall, W. P. Wodchis, and U. Petroz, "Supervisory support, job stress, and job satisfaction among long-term care nursing staff," Journal of Nursing Administration, vol. 37, no. 7-8, pp. 366-372, 2007.

[68] P. A. Parmelee, M. C. Laszlo, and J. A. Taylor, "Perceived barriers to effective job performance among nursing assistants in long-term care," Journal of the American Medical Directors Association, vol. 10, no. 8, pp. 559-567, 2009.

[69] J. Proenca, "Work-family conflict and attitudes of nursing assistants: mediating and moderating effects," Academy of Management Annual Meeting Proceedings, vol. 1, pp. 1-6, 2008.

[70] J. K. Purk and S. Lindsay, "Job satisfaction and intention to quit among frontline assisted living employees," Journal of Housing for the Elderly, vol. 20, no. 1-2, pp. 117-131, 2006.

[71] M. Ramirez, J. A. Teresi, D. Holmes, and S. Fairchild, "Ethnic and racial conflict in relation to staff burnout, demoralization, and job satisfaction in SCUs and non-SCUs," Journal of Mental Health and Aging, vol. 4, no. 4, pp. 459-479, 1998.

[72] B. Resnick, M. Simpson, A. Bercovitz et al., "Testing of the rescare pilot intervention: impact on nursing assistants," Geriatric Nursing, vol. 25, no. 5, pp. 292-297, 2004.

[73] M. Simpson, Factors Related to Job Performance and Job Satisfaction in Certified Nursing Assistants, University of Maryland, Baltimore, Md, USA, 2010.

[74] M. Snow and G. L. White, "What do CNAs want? A survey," Nursing Homes: Long Term Care Management, vol. 56, no. 3, p. 81, 2007.

[75] J. M. Solomon, Influence of leadership on nursing assistants' job satisfaction in a central Maryland nursing home [Ph.D. thesis], University of Phoenix, Tempe, Ariz, USA, 2009.

[76] T. Tannazzo, L. Breuer, S. Williams, and N. A. Andreoli, "A dementia training program to benefit certified nurse assistant satisfaction and nursing home resident outcomes," Alzheimer's Care Today, vol. 9, no. 4, pp. 221-232, 2008.

[77] M. A. Thompson, K. K. Horne, and T. R. Huerta, "Reassessing nurse aide job satisfaction in a Texas nursing home," Journal of Gerontological Nursing, vol. 37, no. 9, pp. 42-49, 2011.

[78] D. A. Tyler, V. A. Parker, R. L. Engle et al., "An Exploration of job design in long-term care facilities and its effect on nursing employee satisfaction," Health Care Management Review, vol. 31, no. 2, pp. 137-144, 2006.

[79] K. A. Walborn, An Analysis of the Relationship between Nursing Assistants' Job Performance and Job Satisfaction in a Nursing Home, George Mason University, 1996.

[80] S. Webb, Testing an Intervention to Decrease (CNA) Turnover, University of Rhode Island, Kingston, RI, USA, 2003.

[81] M. M. Ball, M. L. Lepore, M. M. Perkins, C. Hollingsworth, and M. Sweatman, "They are the reason I come to work': the meaning of resident-staff relationships in assisted living," Journal of Aging Studies, vol. 23, no. 1, pp. 37-47, 2009.

[82] M. G. Bye and J. Iannone, "Excellent care-givers (nursing assistants) of the elderly: what satisfies them about their work," Nursing Homes and Senior Citizen Care, vol. 36, no. 4, pp. 36-39, 1987.

[83] T. X. Karner, R. J. Montgomery, D. Dobbs, and C. Wittmaier, "Increasing staff satisfaction. The impact of SCUs an family involvement," Journal of Gerontological Nursing, vol. 24, pp. 3944, 1998. 
[84] W. Moyle, J. Skinner, G. Rowe, and C. Gork, "Views of job satisfaction and dissatisfaction in Australian long-term care," Journal of Clinical Nursing, vol. 12, no. 2, pp. 168-176, 2003.

[85] Quinn, Factors Influencing Staff Satisfaction in a Long-Term Care Facility, Capella University, 2002.

[86] L. Kmet, R. Lee, and L. Cook, Standard Quality Assessment Criteria for Evaluating Primary Research Papers from a Variety of Fields, Edmonton: Alberta Heritage Foundation for Medical Research, 2004.

[87] K. Khan, G. ter Riet, J. Popay, J. Nixon, J. Kleijnen, and I. I. Satge, "Conducting the review: phase 5 study quality assessment," in Undertaking Systematic Reviews of Research Effectiveness. CDC's Guidance for those Carrying Out or Commissioning Reviews, pp. $1-20$, Centre of Reviews and Dissemination, University of York, 2001.

[88] H. C. W. De Vet, R. A. de Bie, G. J. M. G. Van Der Heijden, A. P. Verhagen, P. Sijpkes, and P. G. Knipschild, "Systematic reviews on the basis of methodological criteria," Physiotherapy, vol. 83, no. 6, pp. 284-289, 1997.

[89] J. J. Deeks, J. Dinnes, R. D’Amico et al., "Evaluating nonrandomised intervention studies," Health Technology Assessment, vol. 7, no. 27, 2003.

[90] N. Jackson and E. Waters, "Criteria for the systematic review of health promotion and public health interventions," Health Promotion International, vol. 20, no. 4, pp. 367-374, 2005.

[91] Critical Appraisal Skills Programme (CASP), Critical Appraisal Skills Programme: Making Sense of Evidence about Clinical Effectiveness, CASP, Cambridge, UK, 2010.

[92] J. Grimshaw, L. M. McAuley, L. A. Bero et al., "Systematic reviews of the effectiveness of quality improvement strategies and programmes," Quality \& Safety in Health Care, vol. 12, no. 4, pp. 298-303, 2003.

[93] M. D. House, Factors that Relate to Job Satisfaction Among Nurse Aides in Long-Term Care Facilities, Colorado State University, 1990. 


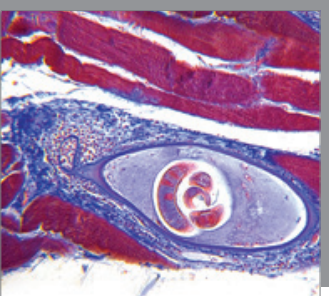

Gastroenterology

Research and Practice
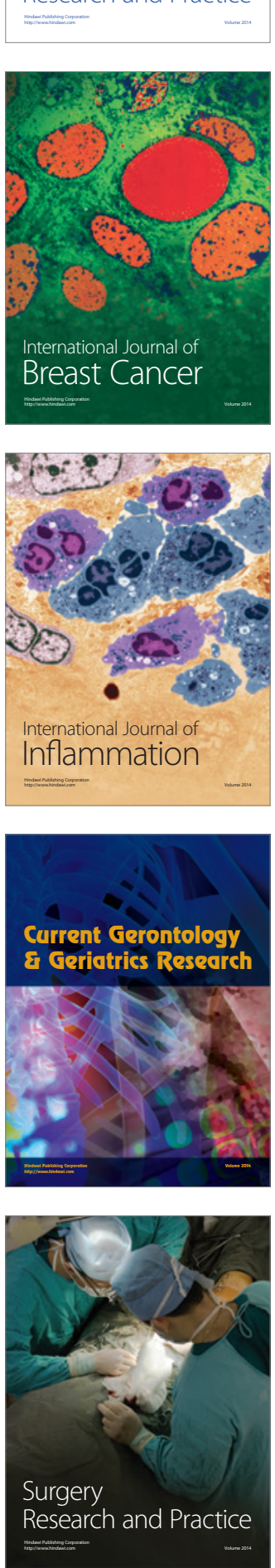

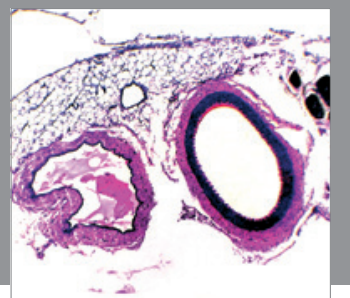

International Journal of Hypertension
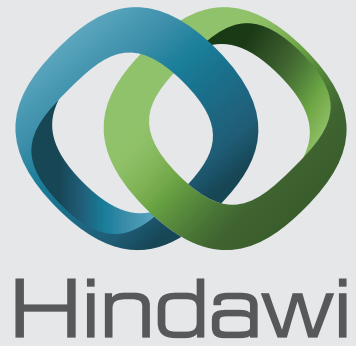

Submit your manuscripts at http://www.hindawi.com
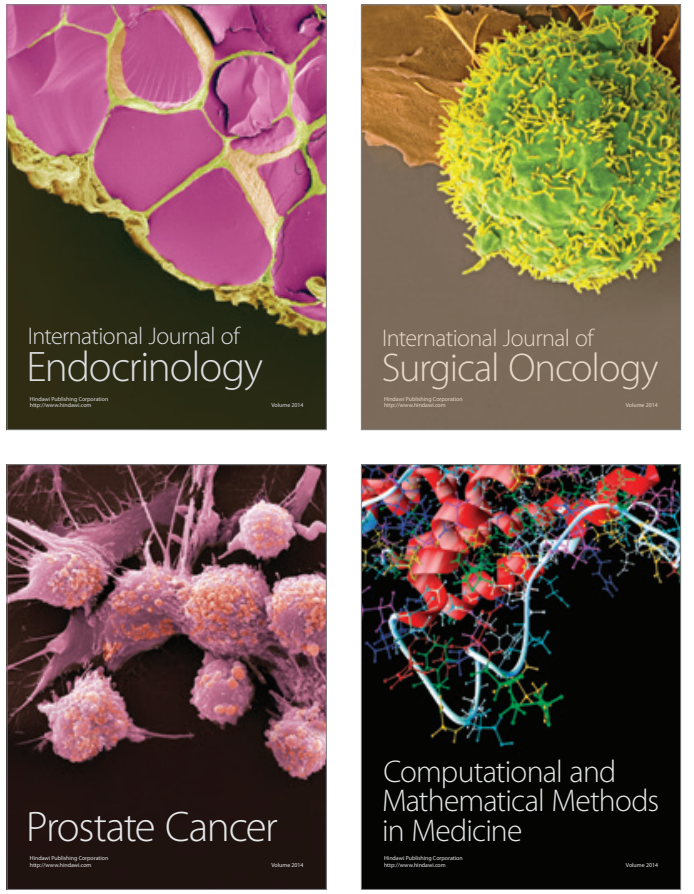
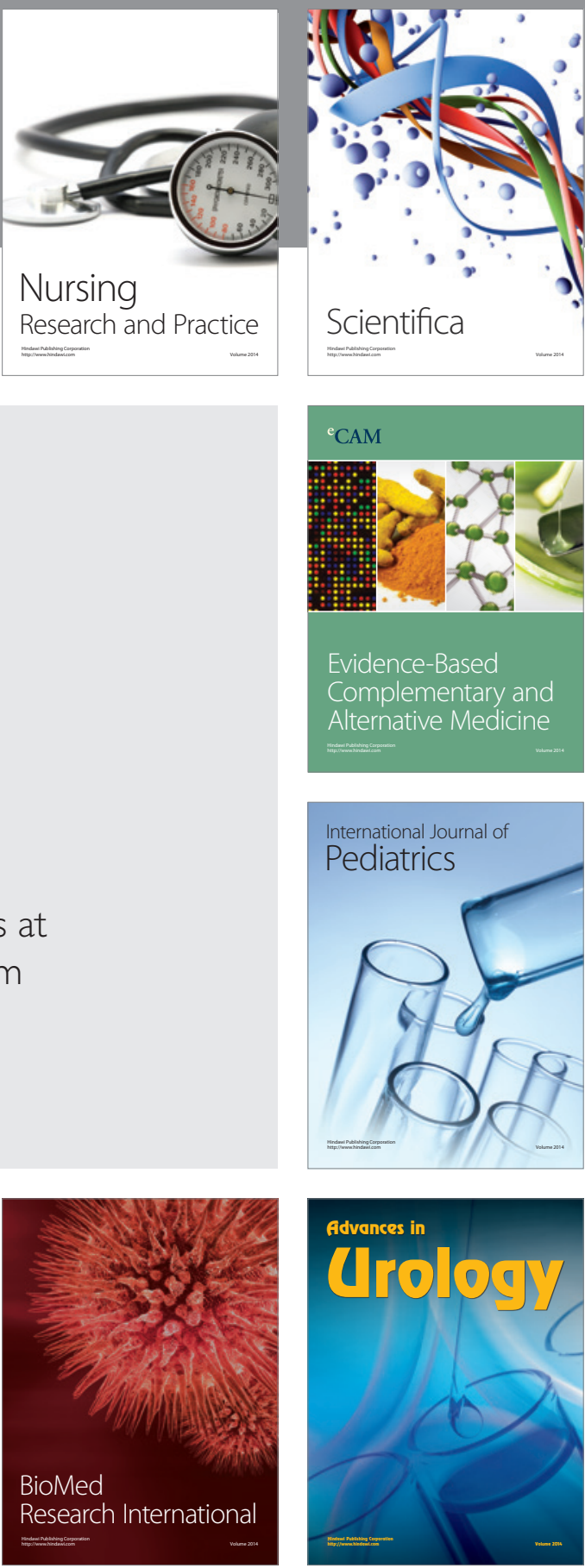

Nursing

Research and Practice

Scientifica

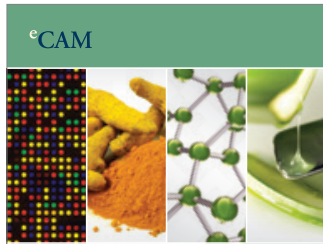

Evidence-Based

Complementary and Alternative Medicine
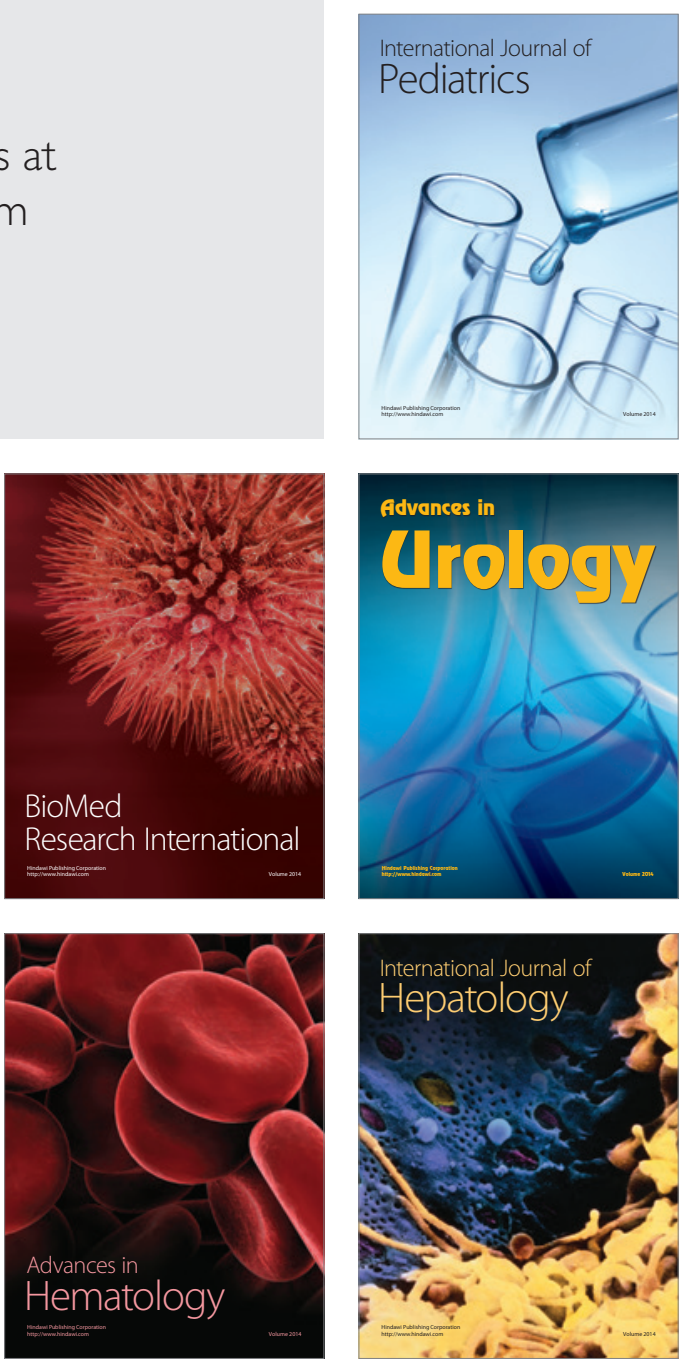